\title{
Crucial role of hematopoietic JAK2 V617F in the development of aortic aneurysms
}

\begin{abstract}
Tetsuro Yokokawa, ${ }^{1,2}$ Tomofumi Misaka, ${ }^{1,3}$ Yusuke Kimishima, ${ }^{1}$ Kento Wada, Keiji Minakawa, ${ }^{4}$ Koichi Sugimoto, ${ }^{1,2}$ Takafumi Ishida, ${ }^{1}$ Soji Morishita, ${ }^{5}$ Norio Komatsu, ${ }^{6}$ Kazuhiko Ikeda ${ }^{4}$ and Yasuchika Takeishi, ${ }^{1}$

${ }^{1}$ Department of Cardiovascular Medicine, Fukushima Medical University, Fukushima; ${ }^{2}$ Department of Pulmonary Hypertension, Fukushima Medical University, Fukushima; ${ }^{3}$ Department of Advanced Cardiac Therapeutics, Fukushima Medical University, Fukushima; ${ }^{4}$ Department of Blood Transfusion and Transplantation Immunology, Fukushima Medical University, Fukushima; ${ }^{5}$ Department of Transfusion Medicine and Stem Cell Regulation, Juntendo University Graduate School of Medicine, Tokyo and ${ }^{6}$ Department of Hematology, Juntendo University Graduate School of Medicine, Tokyo, Japan.
\end{abstract}

\section{ABSTRACT}

TAK2 V617F is the most frequent driver mutation in myeloproliferative neoplasms (MPN) and is associated with vascular complications. However, the impact of hematopoietic JAK2 V617F on aortic aneurysms (AA) remains unknown. Our cross-sectional study indicated that nine $(23 \%)$ of 39 MPN patients with JAK2 V617F exhibited the presence of AA. In order to clarify whether the hematopoietic JAK2 V617F contributes to the AA, we applied bone marrow transplantation (BMT) with the donor cells from Jak2 V617F transgenic (JAK2 ${ }^{\mathrm{V} 617 \mathrm{~F}}$ ) mice or control wild-type (WT) mice into lethally irradiated apolipoprotein E-deficient mice. Five weeks after BMT, the JAK2 ${ }^{\mathrm{V} 617 \mathrm{~F}}$-BMT mice and WT-BMT mice were subjected to continuous angiotensin II infusion to induce AA formation. Four weeks after angiotensin II infusion, the abdominal aorta diameter in the JAK2 $2^{\mathrm{V} 617 \mathrm{~F}}-\mathrm{BMT}$ mice was significantly enlarged compared to that in the WT-BMT mice. Additionally, the abdominal AA-free survival rate was significantly lower in the JAK2 ${ }^{\mathrm{V} 617 \mathrm{~F}}-\mathrm{BMT}$ mice. Hematopoietic JAK2 V617F accelerated aortic elastic lamina degradation as well as activation of matrix metalloproteinase (MMP)-2 and MMP-9 in the abdominal aorta. The numbers of infiltrated macrophages were significantly upregulated in the abdominal aorta of the JAK2 ${ }^{\mathrm{V} 617 \mathrm{~F}}-\mathrm{BMT}$ mice accompanied by STAT3 phosphorylation. The accumulation of BM-derived hematopoietic cells carrying JAK2 V617F in the abdominal aorta was confirmed by use of the reporter green fluorescent proteintransgene. BM-derived macrophages carrying JAK2 V617F showed increases in mRNA expression levels of Mmp2, Mmp9, and Mmp13. Ruxolitinib decreased the abdominal aorta diameter and the incidence of abdominal AA in the JAK $2^{\mathrm{V} 617 \mathrm{~F}}$-BMT mice. Our findings provide a novel feature of vascular complications of AA in MPN with JAK2 V617F.

\section{Introduction}

Myeloproliferative neoplasms (MPN) including polycythemia vera (PV), essential thrombocythemia (ET), and primary myelofibrosis (PMF) are characterized by chronic proliferation of mature myeloid cells and extramedullary hematopoiesis. The prevalence of MPN is reported to be 0.4 to 2.8 per 100,000 persons and the incident rates are 3.1 to 10.9 per one million person-years, which are increasing with age. ${ }^{1,2}$ Major causes of morbidity and mortality in MPN patients are represented by vascular complications, progression to myelofibrosis, and transformation to acute leukemia. ${ }^{3}$ To date, vascular disorders in MPN are known as arterial and venous thrombosis and advanced atherosclerosis. ${ }^{3.5}$ Among the MPN, JAK2 V617F is the most frequent driver mutation, which is observed in over $95 \%$ of PV patients as well as in $50-60 \%$ of ET and PMF patients. ${ }^{6}$ Several murine studies 
have shown that expression of Jak2 V617F confers cytokine-independent growth of the myeloid cells, resulting in a variety of MPN phenotypes, like PV, ET, or PMF depending on the animal model. ${ }^{7,8}$ By contrast, the etiological link between hematopoietic JAK2 V617F and vascular disorders has not fully been elucidated in vivo.

Aortic aneurysms (AA) are a life-threatening aortic disease, characterized by dilatation of the aorta. ${ }^{9}$ There are increasing numbers of patients with AA, with a prevalence in western countries of $1-5 \%$ in the adult population aged over 65 years old. . $^{10,11}$ Although small sized AA remain mostly asymptomatic, AA are slowly progressive, and large AA often lead to aortic rupture and/or sudden death when their diameters are $>6.0 \mathrm{~cm}$ in thoracic AA (TAA) and $>5.0 \mathrm{~cm}$ in abdominal AA (AAA). It has been reported that the incidence of rupture of $\mathrm{AA}$ is 21.3 per 100,000 people in the general population with a mortality of $85-90 \% .^{12}$ Thus, it is important to stratify the risk of AA rupture, and to discover a novel mechanism for the formation and progression of AA. It is known that circulating inflammatory cells play important roles in the development of AA, accompanied by the secretion of various inflammatory factors such as cytokines and chemokines. ${ }^{13,14}$ In this term, inflammatory AAA which characterizes an unusually thickened aneurysm wall and dense adhesions of adjacent intra-abdominal structures represent different features from atherosclerotic AAA. ${ }^{9}$ Although bone marrow (BM) progenitor cells contribute to the tissue inflammation and pathogenesis of cardiovascular diseases such as atherosclerotic diseases, ${ }^{15,16}$ the causal role of BM-derived cells on the onset and progression of AA has not yet been fully explored. Moreover, the prevalence of AA has not been studied in MPN patients with JAK2 V617F, who are more prone to vascular disorders than both the general population and MPN patients with driver mutations other than JAK2 V617F. ${ }^{17}$

In the present study, we hypothesized that hematopoietic JAK2 V617F might be causally associated with the development of AA. Strikingly, we demonstrated that the hematopoietic cells carrying JAK2 V617F promoted AAA progression in mice, as well as a common incidence of $\mathrm{AA}$ in MPN patients with JAK2 V617F.

\section{Methods}

Detailed methods are provided in the Online Supplementary Appendix.

\section{Patients}

We enrolled 39 patients with JAK2 V617F-positive MPN whose computed tomography data were available in a series of our former studies. ${ }^{18,19}$ We evaluated the maximal diameter of the ascending aorta and abdominal aorta via computed tomography imaging. The protocol of the human studies was approved by the Institutional Ethics Committee of the Fukushima Medical University Hospital (approval ID, 29348 and 1242).

\section{Animals}

We used male Jak2 V617F-expressing transgenic (JAK2 ${ }^{\mathrm{V} 617 \mathrm{~F}}$ ) mice.,19 Wild-type (WT) littermates were used as controls. Male apolipoprotein E-deficient (ApoE ${ }^{--}$) mice on a C57BL/6 background were obtained from the Jackson Laboratory (JAX stock number, 002052, Bar Harbor, ME, USA)..$^{20}$ CAG-EGFP reporter mice with a C57BL/6 background were purchased from Japan SLC (Shizuoka, Japan). The JAK2 ${ }^{\mathrm{V} 617 \mathrm{~F}}$ mice were crossed with the CAG-EGFP mice to generate JAK2 ${ }^{\mathrm{V} 617 \mathrm{f}} / \mathrm{CAG-EGFP} \mathrm{double} \mathrm{trans-}$ genic mice (JAK2 $\left.{ }^{\mathrm{V} 617 \mathrm{~F}}-\mathrm{GFP}\right){ }^{21}$ WT littermates were used as controls (WT-GFP). All animal studies were approved by the Fukushima Medical University Animal Research Committee (approval ID, 2019078).

\section{Bone marrow transplantation}

$\mathrm{ApoE}^{-/-}$mice or WT mice aged between 8 and 12 weeks (body weight range, 20-30 g) were lethally irradiated (9.0 Gy) 24 hours before bone marrow transplantation (BMT). ${ }^{19}$ Whole BM cells were harvested from the femurs and tibiae of the mice. BM cells $\left(5.0 \times 10^{6}\right)$ were injected into the $\mathrm{ApoE}^{-/-}$recipient mice or the WT recipient mice via the tail vein.

\section{Angiotensin II-induced aortic aneurysm model}

Five weeks after BMT, the $\mathrm{ApoE}^{-/-}$recipient mice or the WT recipient mice were implanted with an osmotic pump (ALZET micro-osmotic pump MODEL 1004, DURECT Co., Cupertino, CA, USA) ${ }^{22}$ and angiotensin II (Ang II) (1900 ng/kg per min) or saline was continuously infused for 4 weeks. ${ }^{23,24}$

\section{Futher methods}

The details of blood sampling, chimeric analysis, ultrasound imaging and determination of aortic aneurysms, measurements of blood pressure and heart rate, histological analysis, measurement of total cholesterol and triglyceride, gelatin zymography, preparation for BM-derived macrophages, western blot analysis and reverse transcription-quantitative polymerase chain reaction are described in the Online Supplementary Appendix.

\section{Treatment with a JAK1/2 inhibitor}

For in vitro experiments, ruxolitinib (Novartis Pharmaceuticals, Basel, Switzerland) was used at a concentration of $250 \mathrm{nM}$ for 24 hours prior to the RNA extraction. Dimethylsulfoxide was used as a control. For the in vivo study, ruxolitinib dissolved in $0.5 \%$ methylcellulose was administered to the mice orally at $60 \mathrm{mg} / \mathrm{kg}$ twice daily for 4 weeks. ${ }^{25,26}$ We used $0.5 \%$ methylcellulose as a vehicle control.

\section{Statistical analysis}

Data are expressed as mean \pm standard error of the mean (SEM). A value of $P<0.05$ was considered statistically significant.

\section{Results}

\section{Prevalence of aortic aneurysms in patients with myeloproliferative neoplasms}

In order to investigate the incidence of $\mathrm{AA}$ in association with the presence of JAK2 V617F in the hematopoietic cells, we conducted a cross-sectional study that enrolled 39 MPN patients with the JAK2 V617F mutation (mean age, $68 \pm 12$ years; female, $54 \%$ ) in a series of our former studies ${ }^{18,19}$ (Table 1). The allele burden of JAK2 V617F was $51 \pm 30 \%$. Computed tomography revealed the presence of AA in nine (23\%) of the 39 JAK2 V617F-positive MPN patients, ${ }^{9,27}$ including two patients $(22 \%)$ who underwent graft replacement surgery of the aorta due to progression of AA during the MPN course. When we divided the patients into two groups based on the presence of prior histories of thrombotic events as a crucial risk factor in the conventional prognostic system regarding the incidence of arterial and venous thrombotic 
Table 1. Characteristics and prevalence of aortic aneurysms in JAK2 V617F-positive myeloproliferative neoplasm patients.

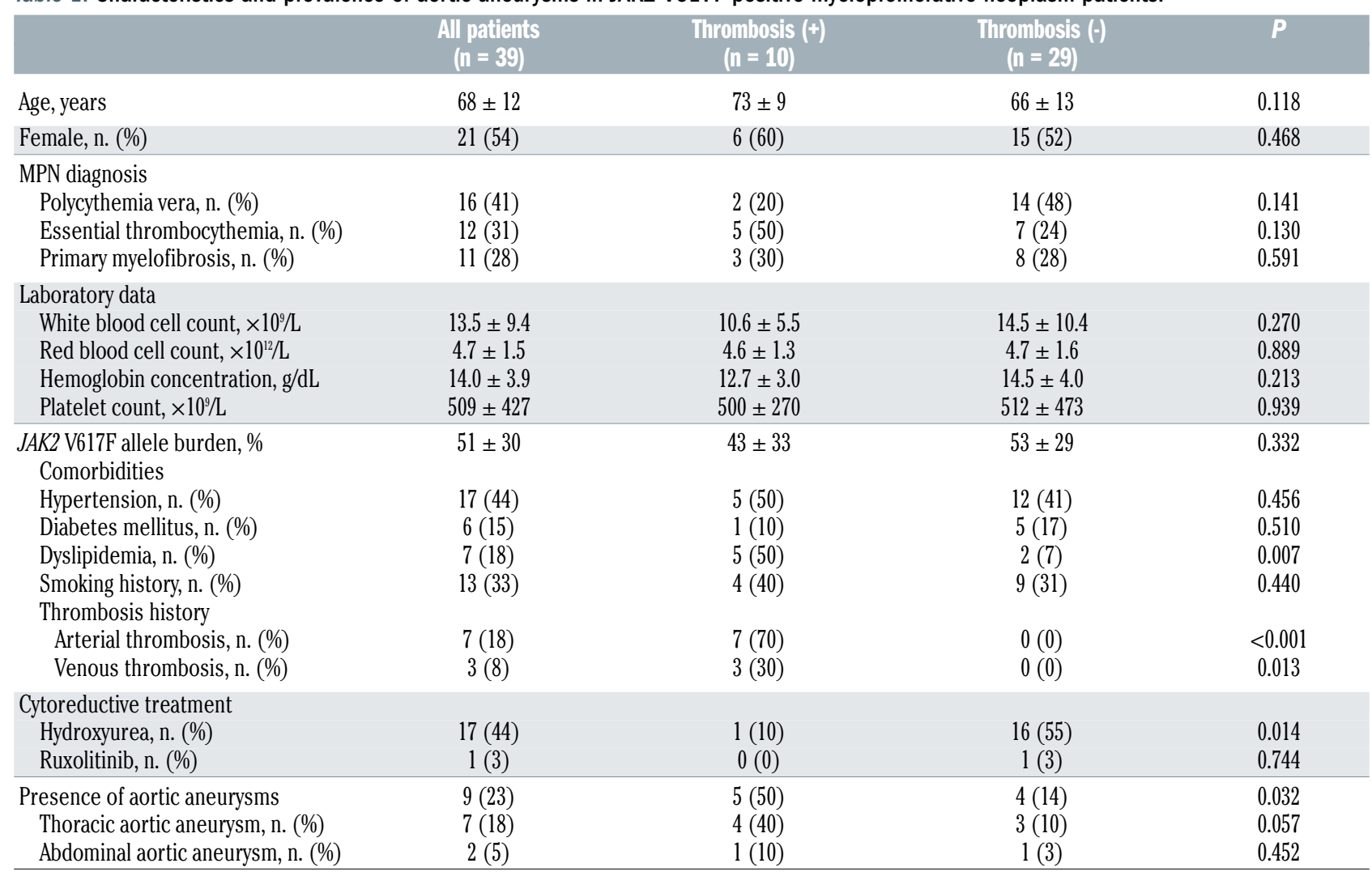

events in PV and $\mathrm{ET}^{28,29}$ the prevalence of AA was significantly higher in the JAK 2V617F-positive MPN patients with a history of thrombosis than in those without (Table 1 and Online Supplementary Table S1). Next, we examined the expression levels of genes known to be related to AA, such as MMP and cytokines, ${ }^{30}$ in circulating leukocytes. The MMP9, which is independently associated with aortic wall thickness and aortic luminal diameter during the process of AA, ${ }^{31}$ as well as TGFB3 and in interleukin 8 (IL8) were significantly increased in JAK2 V617F-positive MPN patients compared to the age- and sex-matched control subjects (Online Supplementary Figure S1 and Online Supplementary Table S2). These findings suggest that incidences of AA are not rare in JAK2 V617F-positive MPN patients, and that the presence of JAK2 V617F in leukocytes may lead to increases in the expression levels of genes involved in the pathogenesis of AA.

\section{Hematopoietic JAK2 V617F promotes abdominal aortic aneurysms formation in angiotensin II-infused ApoE $\mathrm{E}^{-/-}$mice}

In order to clarify whether or not hematopoietic Jak2 V617F could indeed play a mechanistic role in the development of AA, we applied the BMT strategy using the donor BM cells from the JAK2 ${ }^{\mathrm{V} 617 \mathrm{~F}}$ mice ${ }^{7}$ into the lethally irradiated $\mathrm{ApoE}^{-/-}$mice $^{24}\left(\mathrm{JAK}^{\mathrm{V} 617 \mathrm{~F}}-\mathrm{BMT}\right.$ mice) (Figure 1A). The irradiated $\mathrm{ApoE}^{-/-}$recipient mice transplanted with donor BM cells from WT littermates (WT-BMT mice) were used as controls. Five weeks after BMT, the donor cells were similarly engrafted as shown in the chimeric analysis of the peripheral blood cells between the WT-BMT mice and JAK2 ${ }^{\mathrm{V} 617 \mathrm{~F}}$-BMT mice (Figure 1B).
The JAK2 $2^{\mathrm{V} 617 \mathrm{~F}}-\mathrm{BMT}$ mice displayed significantly higher white blood cell counts and spleen weights compared to the WT-BMT mice, indicating a hematological feature of MPN-like phenotype in the JAK2 ${ }^{\mathrm{V} 617 \mathrm{~F}}$-BMT mice (Figure 1C and Online Supplementary Figure S2). Next, the WTBMT mice and JAK2 ${ }^{\mathrm{V} 617 \mathrm{~F}}-\mathrm{BMT}$ mice were continuously infused with Ang II with $1900 \mathrm{ng} / \mathrm{kg} / \mathrm{min}$ for 4 weeks, as a higher dose of Ang II is required to induce the AA formation when the irradiated $\mathrm{ApoE}^{-/-}$recipient mice are used. ${ }^{24}$ At 2 and 4 weeks following Ang II infusion, systolic, mean, and diastolic blood pressures were all significantly increased compared to those of the saline groups, but there were no differences in these parameters between the WT-BMT mice and JAK2 ${ }^{\mathrm{V} 617 \mathrm{~F}}$-BMT mice (Figure 1D; Online Supplementary Figure S3A and S3B). No significant difference was observed regarding the heart rate between the WT-BMT mice and JAK2 ${ }^{\mathrm{V} 617}$-BMT mice after Ang II infusion (Online Supplementary Figure S3C). Total cholesterol and triglyceride concentrations were decreased in the JAK2 ${ }^{\mathrm{V} 617 \mathrm{~F}}-\mathrm{BMT}$ mice compared to WTBMT mice (Online Supplementary Table S3), in agreement with the findings that MPN patients with JAK2 V617F often display low lipid profiles that correlate with advanced disease. ${ }^{32,33}$ Chimerism was not significantly different between the WT-BMT mice and JAK2 ${ }^{\mathrm{V} 617 \mathrm{~F}}$-BMT mice 4 weeks after saline or Ang II infusion (Online Supplementary Figure S3D). We monitored both the thoracic aorta and suprarenal abdominal aorta by ultrasonography. The presence of TAA and AAA was defined as an increase by $\geq 50 \%$ in aortic diameter in comparison to the baseline. We did not observe any significant differences in the thoracic aorta diameter or the incidence of 
A

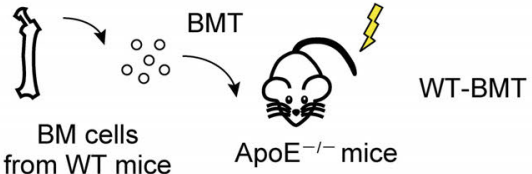

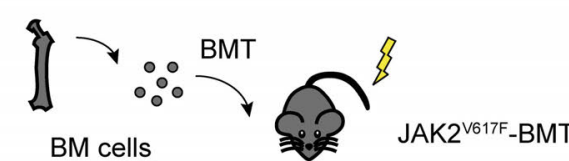
from JAK2 ${ }^{\mathrm{V} 617 \mathrm{~F}}$ mice $\quad \mathrm{ApoE}^{-/-}$mice
B

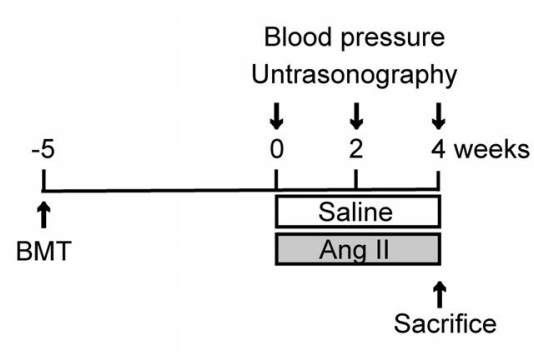

O WT-BMT

JAK2 $2^{\mathrm{V} 617 \mathrm{~F}}-\mathrm{BMT}$

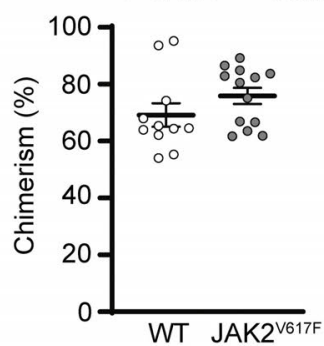

C
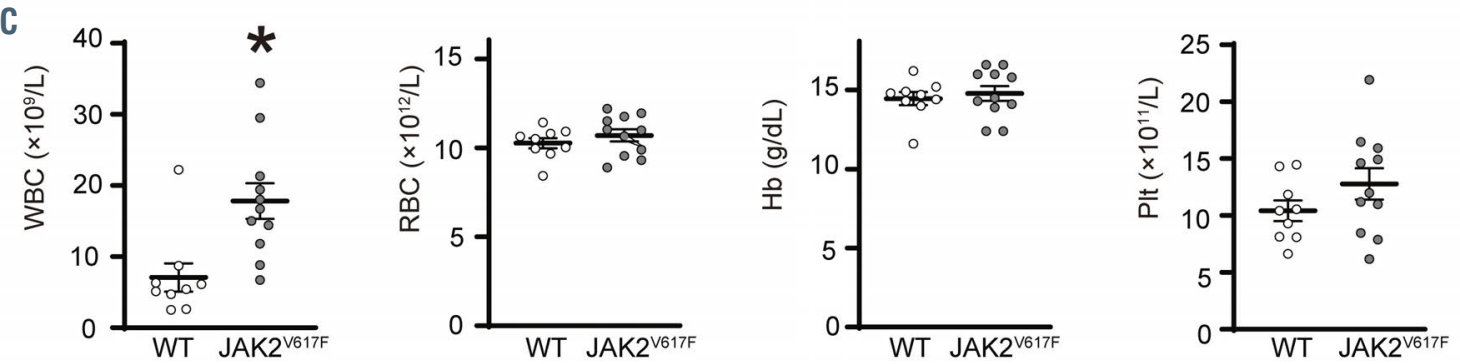

D
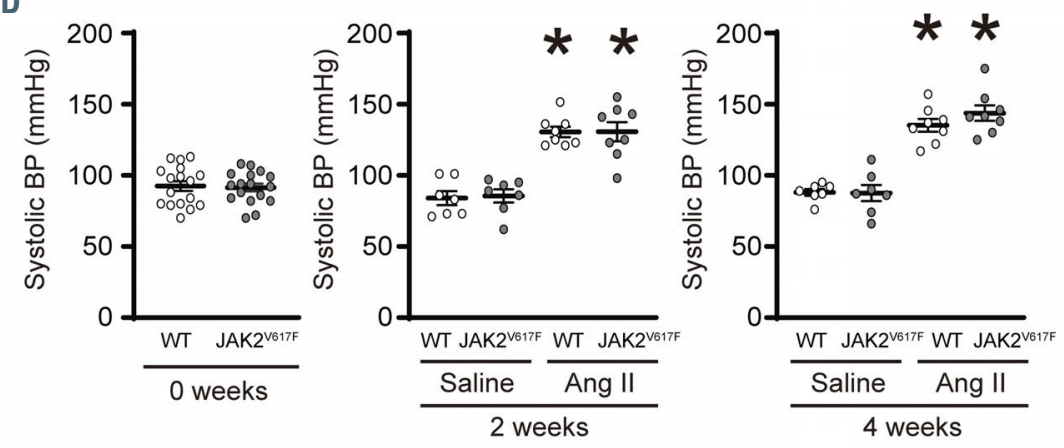

E

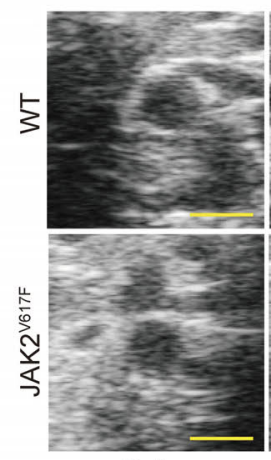

Saline

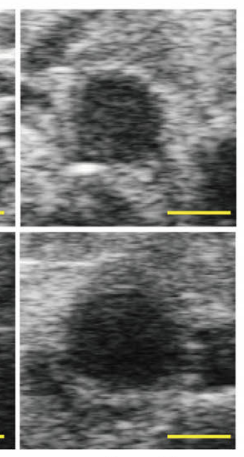

Ang II
$F$

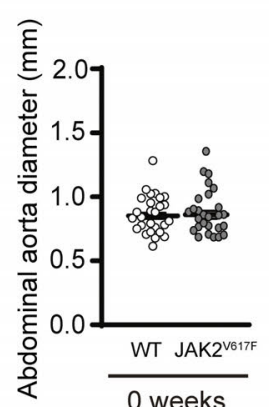

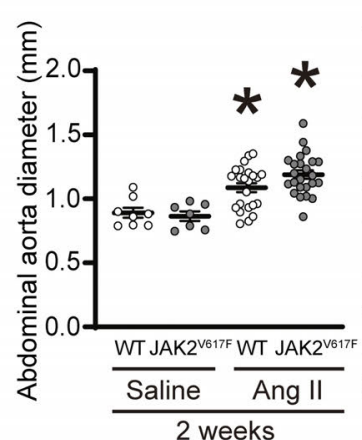

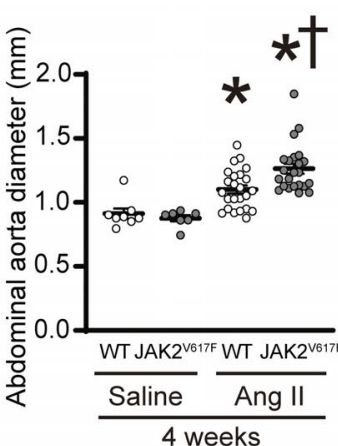

G

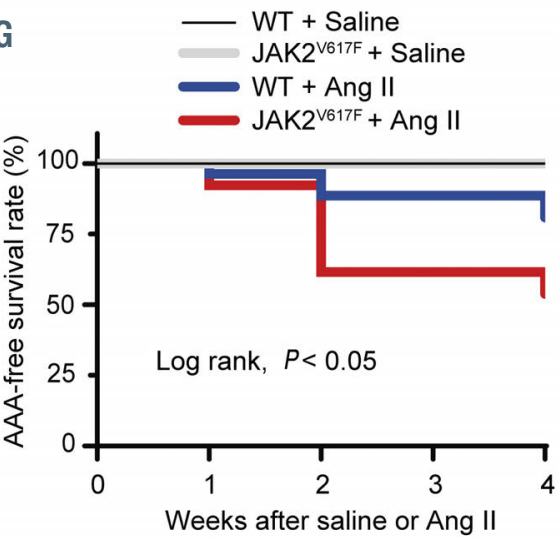

Figure 1. Hematopoietic JAK2 V617F contributes to abdominal aortic aneurysm formation. (A) Schematic diagram of the experimental design of bone marrow (BM) transplantation (BMT). BM cells from control wild-type (WT) mice or JAK2 $2^{\mathrm{v} 617 \mathrm{~F}}$ mice were injected into the lethally irradiated ApoE ${ }^{-/-}$recipient mice. Five weeks after BMT, the $\mathrm{ApoE}^{-/-}$recipient mice transplanted with WT BM cells (WT-BMT mice) or JAK2 ${ }^{\mathrm{v} 617 \mathrm{~F}} \mathrm{BM}$ cells (JAK2 ${ }^{\mathrm{v} 617 \mathrm{~F}}$-BMT mice) were subjected to saline or angiotensin II (Ang II, $1900 \mathrm{ng} / \mathrm{kg}$ per min) infusion for 4 weeks. (B) Chimerism of donor cells in the peripheral leukocytes $(n=11-13)$ and $(C)$ blood cell counts $(n=9-11)$ in the WT-BMT mice and JAK ${ }^{2 v 617}$-BMT mice at 5 weeks after BMT. ${ }^{*} P<0.05$ versus the WT-BMT mice by the unpaired Student's $t$-test. (D) Systolic blood pressure (BP) at the baseline ( $n=17$ each), 2 weeks $(n=7-10)$ and 4 weeks $(n=7-8)$ after saline or Ang II infusion. (E) Representative ultrasound images of abdominal aorta under saline or Ang II infusion for 4 weeks. Scale bars, $1.0 \mathrm{~mm}$. (F) Abdominal aorta diameter at the baseline $(n=33-34), 2$ weeks $(n=7-24)$ and 4 weeks $(n=7-24)$ after saline or Ang II infusion. (G) Abdominal aortic aneurysm (AAA)-free survival rate in the WT-BMT mice and JAK2 ${ }^{\mathrm{v} 617 \mathrm{f}}$-BMT mice after saline or Ang II infusion ( $\mathrm{n}=7-26$ ) by log-rank test. All data are presented as mean \pm standard error of the mean. ${ }^{*} P<0.05$ vs. the corresponding saline-infused mice and ${ }^{\dagger} P<0.05$ vs. the corresponding WT-BMT mice by one-way ANOVA with Tukey post-hoc analysis. WT, ApoE ${ }^{-/}$: mice transplanted with bone marrow cells from wild-type mice; JAK2 $2^{\mathrm{v} 617 \mathrm{f}}$, ApoE ${ }^{-/}$: mice transplanted with bone marrow cells from JAK2 ${ }^{\mathrm{V} 617 \mathrm{~F}}$ mice; Ang II: angiotensin II; WBC: white blood cell count; RBC: red blood cell count; Hb: hemoglobin concentration; Plt: platelet count. 
TAA between the WT-BMT mice and JAK2 ${ }^{\mathrm{V} 617 \mathrm{~F}}-\mathrm{BMT}$ mice after Ang II infusion (Online Supplementary Figure S4). In contrast, although abdominal aorta diameters in both WT-BMT mice and JAK2 ${ }^{\mathrm{V} 617 \mathrm{~F}}$-BMT mice were significantly increased at 2 and 4 weeks after Ang II infusion compared to the corresponding saline-infused groups, the abdominal aorta diameter in the JAK2 $2^{\mathrm{V} 617 \mathrm{~F}}$-BMT mice was significantly enlarged compared to that of WT-BMT mice at 4 weeks after Ang II infusion (Figure 1E and 1F). The incidence of AAA was $0 \%$ in both the saline-infused WT-BMT mice and JAK2 ${ }^{\mathrm{V} 617}-\mathrm{BMT}$ mice, while it was $18.5 \%$ of the WT-BMT mice (5 of 27 ) and $46.1 \%$ of the JAK2 $2^{\mathrm{V} 61 \mathrm{~F}}-\mathrm{BMT}$ mice (12 of 26) developed AAA after Ang II infusion. Accordingly, Kaplan-Meier analysis demonstrated that the AAA-free survival rate was significantly lower in the JAK2 $2^{\mathrm{V} 17 \mathrm{~F}}$-BMT mice than in WT-BMT mice (Figure 1G). No death was observed in either salineinfused WT-BMT mice or JAK2 ${ }^{\mathrm{V} 17 \mathrm{~F}}-\mathrm{BMT}$ mice, while $3.7 \%$ of the Ang II-infused WT-BMT mice (1 of 27) and $7.6 \%$ of the Ang II-infused JAK2 ${ }^{\mathrm{V} 617 \mathrm{~F}}-\mathrm{BMT}$ mice (2 of 26 ) died from AAA rupture during the study period. Although continuous Ang II infusion significantly increased heart weight in both the WT-BMT and the JAK2 ${ }^{\mathrm{V} 617 \mathrm{~F}}-\mathrm{BMT}$ mice, there was no significant difference between the two groups (Online Supplementary Figure S2B). When we used mice with a WT background as recipients, as opposed to the $\mathrm{ApoE}^{-/}$recipient mice, the WT recipients transplanted with JAK2 $2^{\mathrm{V} 617 \mathrm{~F}} \mathrm{BM}$ cells did not exhibit AA formation (Online Supplementary Figure S5). These findings indicate that JAK2 V617F-mediated AAA development is required for an ApoE-deficient background experimentally, which is consistent with the previous work that the incidence of AAA in WT mice was much lower than in hyperlipidemic mice. ${ }^{34}$ Taken together, these data suggest that hematopoietic JAK2 V617F promoted the formation of AAA in response to continuous Ang II infusion independently of the levels of elevated blood pressure.

\section{Hematopoietic JAK2 V617F induces aortic elastic lamina degradation and rupture accompanied by activation of matrix metalloproteinases}

In the morphometric analysis of aortas, a local enlargement of the abdominal aorta with a blood clot was observed in the Ang II-infused JAK2 ${ }^{\mathrm{V} 117}$-BMT mice (Figure 2A). Hematoxylin-eosin staining indicated thickening of the arterial wall as well as inflammatory cell infiltration accompanied by thrombus formation in the JAK2 $2^{\mathrm{V} 17 \mathrm{~F}}-\mathrm{BMT}$ mice after Ang II infusion (Figure 2B). Elastica-Masson staining revealed that discontinuity and breakage of elastin fibers in the aortic wall were detected in the Ang II-infused JAK2 ${ }^{\mathrm{V} 617 \mathrm{~F}}$-BMT mice (Figure 2B). The degree of elastin disruption in the abdominal aortic medial layers was more severe in the JAK2 $2^{\mathrm{V} 617 \mathrm{~F}} \mathrm{BMT}$ mice than in WT-BMT mice after Ang II infusion based on the quantitative analysis by grading scores ${ }^{35}$ (Figure 2C). Activation of MMP, particularly MMP-2 and MMP-9, is required to produce AAA. ${ }^{36}$ Gelatin zymography assay using homogenates from the abdominal aorta demonstrated that either MMP-2 activity or pro MMP-9 expression levels were not different between saline-infused WT-BMT and JAK2 ${ }^{\mathrm{V} 617}$-BMT mice while both MMP-2 activity and pro MMP-9 expression were significantly elevated in the JAK2 $2^{\mathrm{V} 617 \mathrm{~F}}$-BMT mice compared to WT-BMT mice after Ang II infusion (Figure 2D and $2 \mathrm{E}$ ). These data suggest that hematopoietic JAK2
V617F contributes to elastin disruption accompanied by activation of MMP, resulting in enlargement of the abdominal aorta and AAA rupture.

\section{Inflammatory responses are accelerated in the abdominal aortas in JAK2 ${ }^{\text {v617F-bone marrow }}$ transplantation mice with activation of STAT3}

We next examined inflammatory responses in the AAA formation and progression. ${ }^{37}$ Immunohistochemical analysis showed that there were significant increases in CD $45^{+}$leukocytes, $\mathrm{CD}^{6} 8^{+}$macrophages, Ly6B. $2^{+}$neutrophils, and TER $119^{+}$erythrocytes in the abdominal arterial walls in the JAK2 ${ }^{\mathrm{V} 617 \mathrm{~F}}-\mathrm{BMT}$ mice compared to WTBMT mice after Ang II infusion (Figure 3A and 3B; Online Supplementary Figure S6). The phosphorylation levels of STAT3 in the abdominal aorta were not significantly different between saline-infused WT-BMT mice and JAK2 $2^{\mathrm{V} 617 \mathrm{~F}}-\mathrm{BMT}$ mice, but the levels in the JAK2 $2^{\mathrm{V} 617 \mathrm{~F}}-\mathrm{BMT}$ mice were higher than those in the WT-BMT mice in response to Ang II (Figure 4A). For the assessment of the inflammatory mediators related to AAA development, mRNA expression levels of Cclo, an important macrophage chemokine, as well as Tgfb1, which is involved in collagen and elastin production, were increased in the abdominal aorta of the JAK2 $2^{\mathrm{V} 617 \mathrm{~F}}-\mathrm{BMT}$ mice in comparison to the levels in the WT-BMT mice after Ang II infusion. These results suggest that macrophages and neutrophils carrying JAK2 V617F induce inflammatory responses in abdominal aortic walls in response to Ang II.

\section{Characterization of bone marrow-derived \\ hematopoietic cells carrying JAK2 V617F in the abdominal aorta by use of GFP-transgene}

In order to further characterize the specific contribution of BM-derived circulating cells in the AAA formation in the Ang II-infused JAK2 $2^{\mathrm{V} 617 \mathrm{~F}}$-BMT mice, we generated double transgenic mice by crossing the JAK2 $2^{\mathrm{V} 617 \mathrm{~F}}$ mice with CAG-EGFP mice (JAK2 $\left.{ }^{\mathrm{V} 617 \mathrm{~F}}-\mathrm{GFP}\right){ }^{21}$ Then, we transplanted BM cells derived from JAK2 ${ }^{\mathrm{V} 617}$-GFP mice or control WT-GFP mice into lethally irradiated $\mathrm{ApoE}^{-/-}$mice. After BMT followed by Ang II infusion for 4 weeks, immunostaining showed that more GFP+ cells accumulated in the aorta of the JAK2 ${ }^{\mathrm{V} 617 \mathrm{~F}}-\mathrm{GFP}-\mathrm{BMT}$ mice compared to WT-GFP BMT mice (Figure 5A). In the JAK2 $2^{\mathrm{V} 617 \mathrm{~F}}$ GFP-BMT mice, most GFP ${ }^{+}$cells expressed CD45, CD68, or Ly6B.2 in the abdominal aorta (Figure 5B). These data indicate that the accumulated inflammatory cells carrying JAK2 V617F are mobilized from BM, migrated, and engrafted into the aortic walls in response to Ang II stimulation.

\section{Impact of bone marrow-derived JAK2 ${ }^{\mathrm{V} 617 \mathrm{~F}}$ macrophages on the genesis of matrix metalloproteinase}

We next cultured mononuclear cells isolated from the BM in the presence of granulocyte-macrophage colony-stimulating factor to elucidate the effect of JAK2 ${ }^{\mathrm{V} \text { Vir }}$ macrophages on MMP. The BM cells were mostly differentiated into macrophages with proportions of $\mathrm{CD}^{+} 8^{+}$cells of over $80 \%$ both in the WT and JAK2 ${ }^{\mathrm{V} 61 \mathrm{~F}} \mathrm{BM}$-derived cells (Figure 6A). The mRNA expression levels of Mmp2 and Mmp 9 were significantly increased in the JAK2 $2^{\mathrm{V} 617 \mathrm{~F}}$ BM-derived macrophages compared to the WT BM-derived macrophages (Figure 6B). Additionally, the expression levels of $M m p 13$, an activator of MMP-9, ${ }^{38}$ in the JAK2 ${ }^{\mathrm{V} 117 \mathrm{~F}} \mathrm{BM}$ - 
A
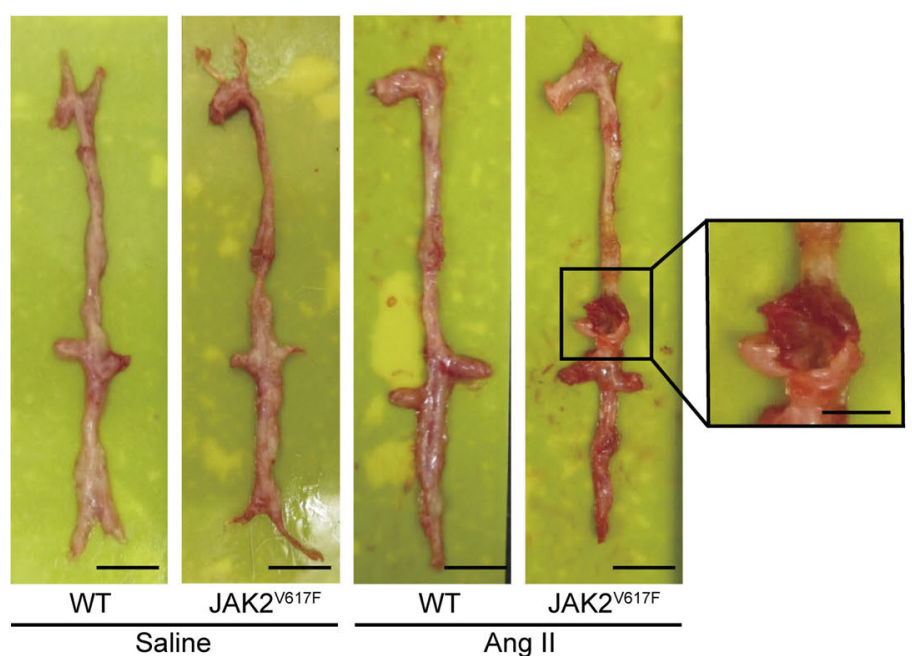

B
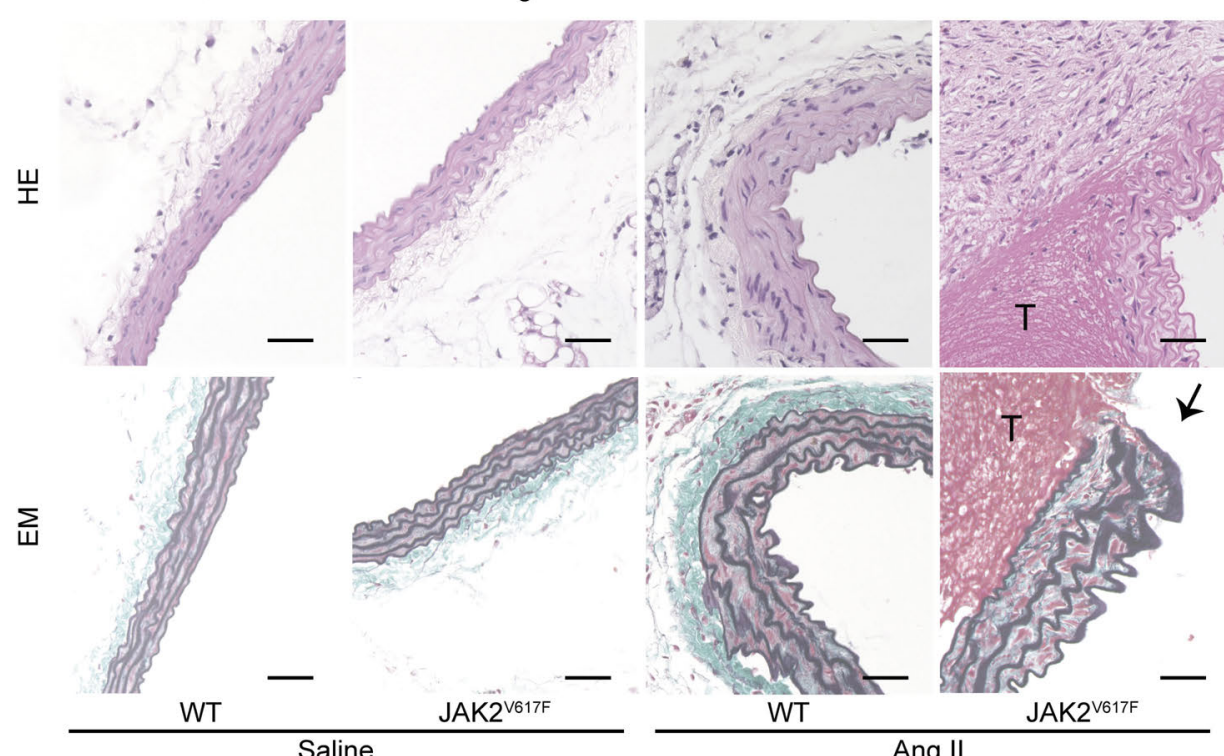

D

C

\section{$\square$ WT-BMT}

JAK2 ${ }^{\mathrm{V} 17 F}-\mathrm{BMT}$

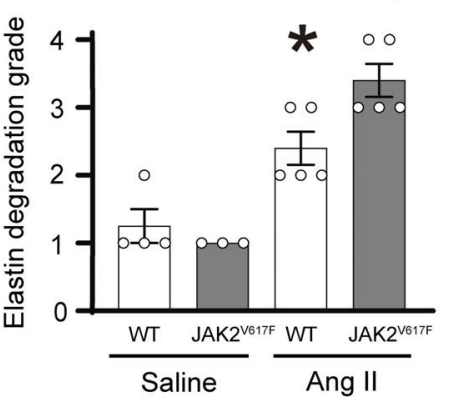

$\mathrm{E}$

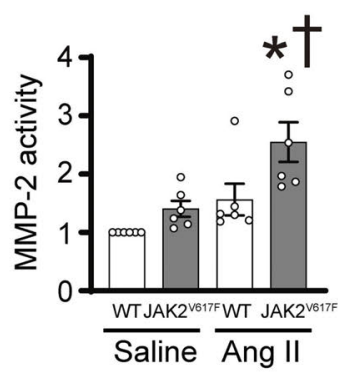

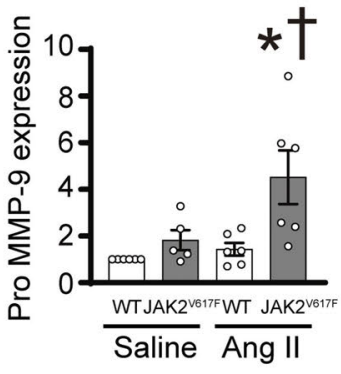

Pro MMP-9-

Pro MMP-2MMP-2-

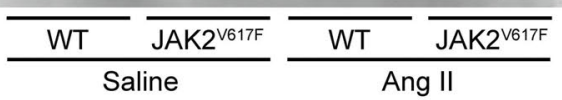

Figure 2. Hematopoietic JAK2 V617F induces elastin degradation and rupture of aortic aneurysm accompanied by matrix metalloproteinase activation. (A) Representative images of the aorta from the WT-BMT mice and JAK2 ${ }^{\mathrm{V} 617 \mathrm{~F}}$-BMT mice 4 weeks after saline or Ang II or infusion. Scale bars, $5 \mathrm{~mm}$. The boxed area from the Ang II-infused JAK2 ${ }^{\mathrm{v} 617 \mathrm{~F}}$-BMT mice was highlighted at higher magnification. Scale bar, $2.5 \mathrm{~mm}$. (B) Representative images of hematoxylin-eosin (HE)- and Elastica-Masson (EM)-stained sections. 'T' indicates thrombus in the aorta. Black arrow indicates the rupture site of the aorta. Scale bars, $50 \mu \mathrm{m}$. (C) Quantitative analysis of degradation grade in EM-stained sections ( $n=3-5)$. (D) A typical image of gelatin zymography using homogenates from abdominal aortas of the WT-BMT mice and JAK2 ${ }^{\text {V617F-BMT }}$ mice 4 weeks after saline or Ang II infusion. (E) Densitometric analysis of the gelatin zymography ( $n=5-6)$. The sum of matrix metalloproteinase (MMP)-2 and pro MMP-2 bands was evaluated as MMP-2 activity. All data are presented as mean \pm standard error of the mean. ${ }^{*} P<0.05$ vs. the corresponding saline-infused mice and ${ }^{\dagger} P<0.05$ vs. the Ang II-infused WT-BMT mice by one-way ANOVA with Tukey post-hoc analysis. $\mathrm{WT}, \mathrm{ApoE}^{---}$: mice transplanted with bone marrow (BM) cells from wild-type (WT) mice; $\mathrm{JAK} 2^{\mathrm{V} 617 \mathrm{~F}}, \mathrm{ApoE}^{---}$: mice transplanted with $\mathrm{BM}$ cells from JAK2 ${ }^{\mathrm{V} 617 \mathrm{~F}}$ mice; BMT: bonemarrow transplantation; Ang II: angiotensin II. 
derived macrophages were higher than those in the WT BM-derived macrophages (Figure 6B). Ruxolitinib, a selective JAK1/2 inhibitor, significantly decreased both Mmp2 and Mmp9 expression levels in the JAK2 $2^{\mathrm{V} 617 \mathrm{~F}}$ macrophages (Figure 6C). These data suggest that BM-derived macrophages carrying JAK2 V617F play important roles in the genesis of MMP.
Inhibition of JAK1/2 with ruxolitinib prevents hematopoietic JAK2 V617F-induced abdominal aortic aneurysm formation

We investigated whether inhibition of JAK1/2 could attenuate hematopoietic JAK2 V617F-induced AAA formation (Figure 7A). Ruxolitinib treatment significantly decreased white blood cell counts and spleen weights in

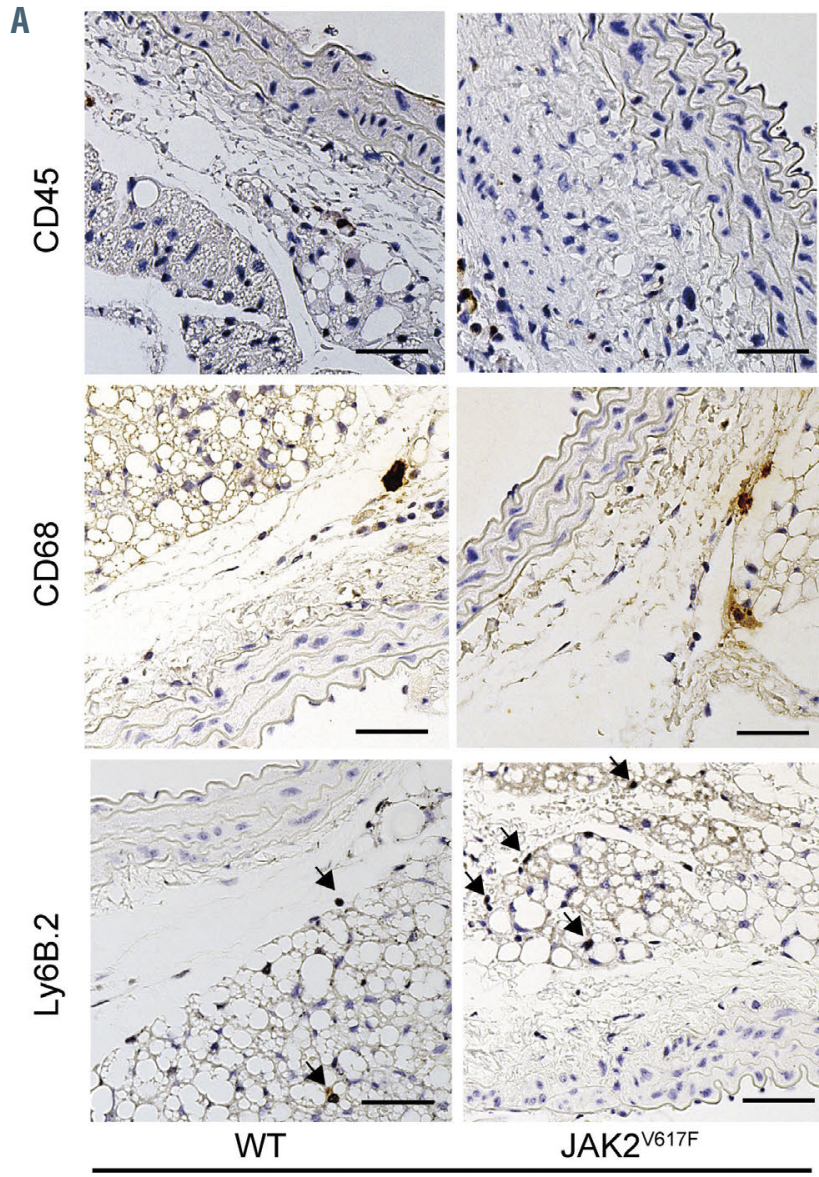

Saline

B

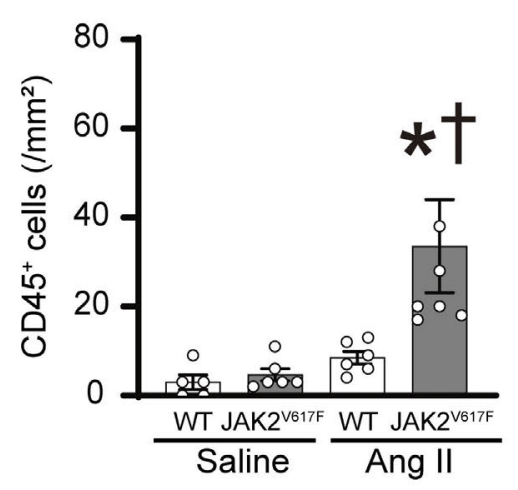

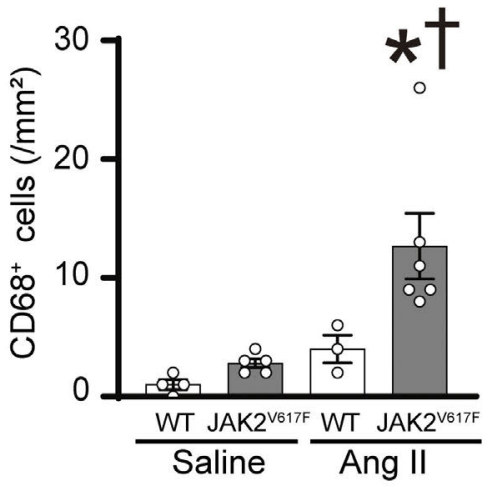

WT-BMT

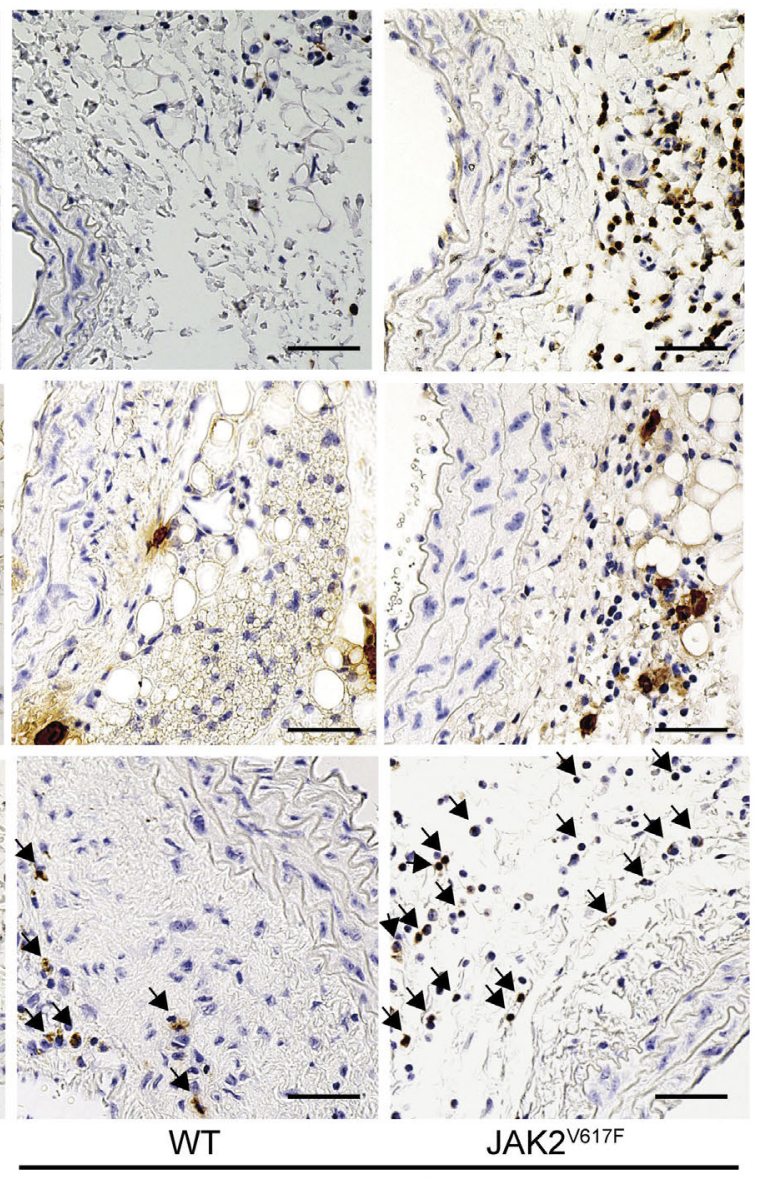

Ang II

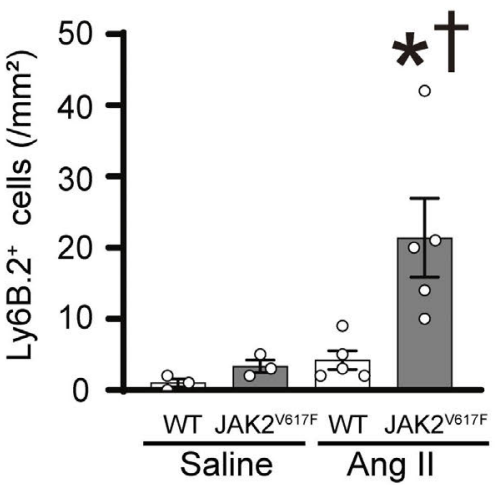

Figure 3. Hematopoietic JAK2 V617F increases inflammatory cell infiltration in the abdominal aorta. (A) Representative immunohistochemical images of the aorta stained by anti-CD45, CD68, and Ly6B.2 antibodies in the WT-BMT mice and JAK2 $2^{\mathrm{V} 617 \mathrm{~F}}$-BMT mice 4 weeks after saline or angiotensin II infusion. Arrows indicate Ly6B.2-positive cells. Scale bars, 50 um. (B) Quantitative analyses of the $\mathrm{CD}_{4} 5^{+}(n=5-6), \mathrm{CD}^{+}(\mathrm{n}=3-5)$, and Ly6B.2 ${ }^{+}(n=3-5)$ cells in the aortic sections. All data are presented as mean \pm standard error of the mean. ${ }^{*} P<0.05$ vs. the corresponding saline-infused mice and ${ }^{\dagger} P<0.05$ vs. the Ang II-infused WT-BMT mice by one-

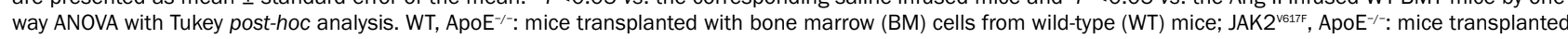
with BM cells from JAK2 ${ }^{\mathrm{V} 617 \mathrm{f}}$ mice; BMT: BM transplantation; Ang II: angiotensin II. 
the Ang II-infused JAK2 ${ }^{\mathrm{V} 617 \mathrm{~F}}$-BMT mice (Figure 7B; Online Supplementary Figure $7 A$ ), but did not affect the levels of blood pressures or heart rate (Figure 7C; Online Supplementary Figure $S 7 B$ to $D$ ). Total cholesterol and triglyceride concentrations were increased in the ruxolitinib-treated JAK2 $2^{\mathrm{V} 617 \mathrm{~F}}$-BMT mice compared to the vehicle-treated JAK2 ${ }^{\mathrm{V} 617 \mathrm{~F}}$-BMT mice, as previously reported in MPN patients (Online Supplementary Table S5). Importantly, abdominal aorta diameters were significantly decreased in the ruxolitinib-treated JAK2 $2^{\mathrm{V} 617 \mathrm{~F}}-\mathrm{BMT}$ mice compared to the vehicle-treated JAK2 ${ }^{\mathrm{V} 617 \mathrm{~F}}$-BMT mice at both 2 and 4 weeks after Ang II infusion (Figure $7 \mathrm{D}$ and $7 \mathrm{E})$. The incidence of AAA was $64 \%$ of the vehicle-treated JAK2 ${ }^{\mathrm{V} 617 \mathrm{~F}}$-BMT mice (7 of 11 ) while $18 \%$ of the ruxolitinib-treated JAK2 ${ }^{\mathrm{V} 617 \mathrm{~F}}$-BMT mice (2 of 11 ). Accordingly, the Kaplan-Meier analysis demonstrated that the AAA-free survival rate was significantly higher in the ruxolitinib-treated JAK2 $2^{\mathrm{V} 617 \mathrm{~F}}$-BMT mice than in the vehicle-treated JAK2 ${ }^{\mathrm{V} 617 \mathrm{~F}}-\mathrm{BMT}$ mice (Figure 7F). Ruxolitinib decreased MMP-2 activity and pro MMP-9 expression in the abdominal aorta in Ang II-infused JAK2 ${ }^{\mathrm{V} 617 \mathrm{~F}}$-BMT mice (Figure 7G). In contrast, thoracic aorta diameters or incidence of TAA were not different between the ruxolitinib- and vehicle-treated JAK2 ${ }^{\mathrm{V} 617 \mathrm{~F}}$ BMT mice (Online Supplementary Figure S7E and S7F). Taken together, these findings strongly suggest that hematopoietic JAK2 V617F plays a causal role in the onset and development of AAA.

\section{Discussion}

The present study is the first to demonstrate that hematopoietic cells carrying JAK2 V617F are causally associated with AAA formation in mice. In response to Ang II stimulation, the infiltrated $\mathrm{CD}^{+} 8^{+}$macrophages with JAK2 V617F in the arterial walls may have contributed to the genesis and activation of matrix metalloproteinase, resulting in increases of elastin degradation in the abdominal aorta and the development of AAA. Our findings provide a novel feature of vascular complication of $\mathrm{AA}$ in MPN patients due to the constitutive activation of the hematopoietic JAK-STAT pathway.

To date, vascular complications of MPN patients have been reported mainly in the arterial and venous thrombotic events from clinical and murine studies. ${ }^{3,39}$ History of thrombosis and age $>60$ years are the highest risk factors for thrombosis in MPN patients. ${ }^{40}$ Interestingly, JAK2 V617F-positive MPN patients with a history of thrombosis more frequently showed AA, suggesting that history of thrombosis may be a risk factor for AA as well as thrombosis, whereas age was not different between JAK2 V617F-positive MPN patients with AA and those without AA. Leukocytosis is another factor that increases the risks of thrombosis in MPN patients whereas the roles of increased number or functional alteration of platelets have been controversial in cases of thrombosis in MPN, ${ }^{3,41}$ indicating that abnormal MPN-clone-derived leukocytes

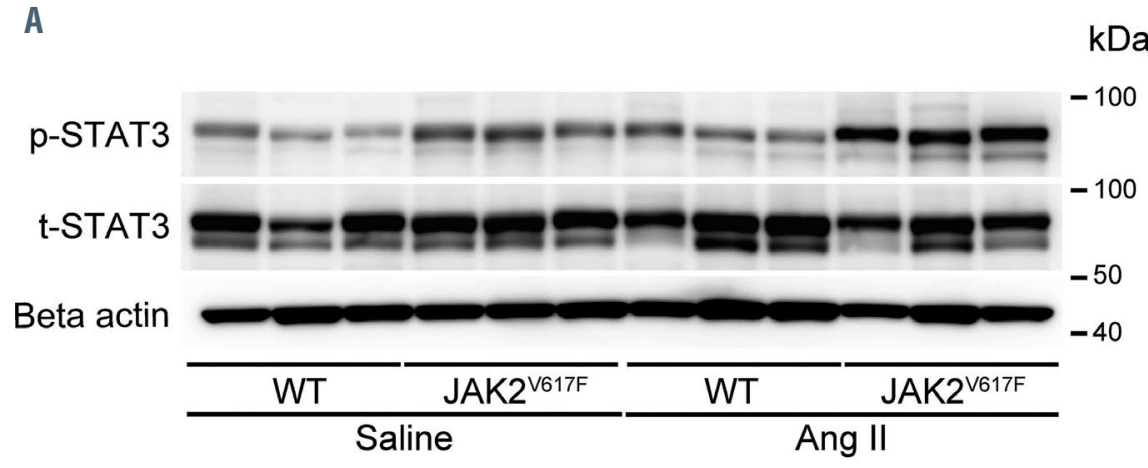

B

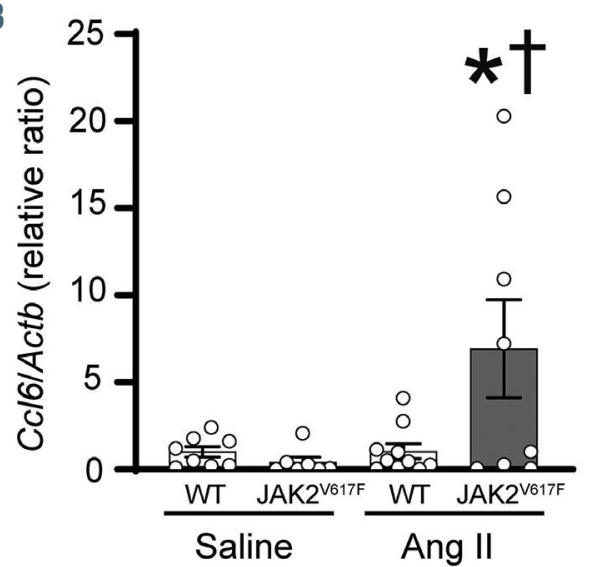

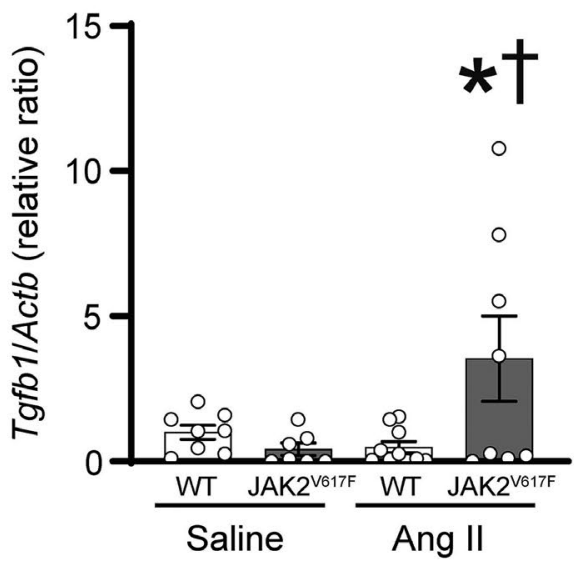

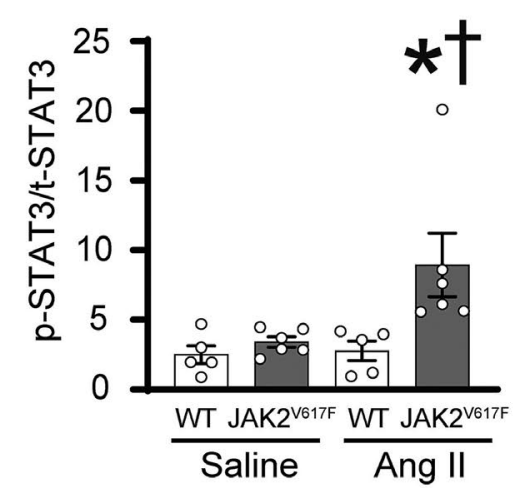

Figure 4. Hematopoietic JAK2 V617F increases STAT3 phosphorylation and cytokine expression in response to angiotensin II infusion in the abdominal aorta. (A) Western blot analysis on the STAT3 in the abdominal aorta. Aorta extracts from the WT-BMT mice or JAK2 ${ }^{\mathrm{v} 6175}$-BMT mice were immunoblotted with the indicated antibodies. Phosphorylated STAT3 (p-STAT3) to total STAT3 (t-STAT3) ratios are shown in the bar graphs. The average value for the saline-infused WT-BMT mice was set to 1 ( $n=5-6)$. $\beta$ actin was used as the loading control. (B) Relative mRNA expression levels of Ccl 6 and Tgfb1 in the aorta. Actb was used for normalization. The average value for saline-infused WT-BMT mice was set to $1(n=7-10)$. All data are presented as mean \pm standard error of the mean. $* P<0.05$ vs. the corresponding saline-infused mice and ${ }^{\dagger} P<0.05$ vs. the Ang II-infused WT-BMT mice by one-way ANOVA with Tukey post-hoc analysis. WT, ApoE ${ }^{-/}:$mice transplanted with bone marrow (BM) cells from wild-type (WT) mice; JAK2 ${ }^{\mathrm{V} 617 \mathrm{~F}}, \mathrm{ApoE}^{-/-}$: mice transplanted with BM cells from JAK2 ${ }^{\mathrm{v} 617 \mathrm{~F}}$ mice; BMT: BM transplantation; Ang II: angiotensin II. 
A

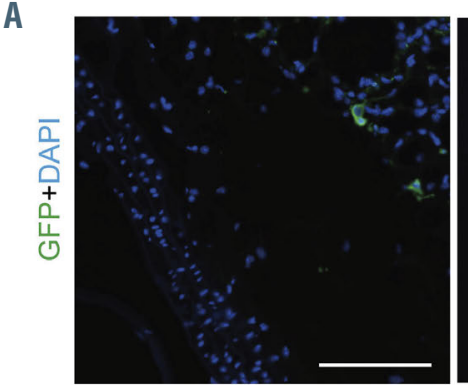

WT-GFP

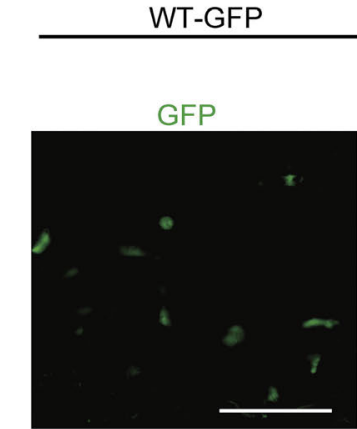

GFP

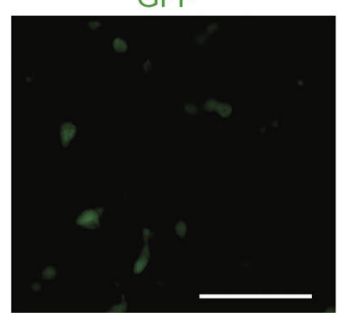

GFP

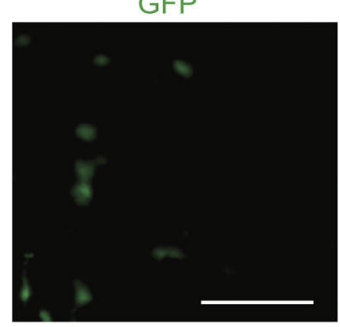

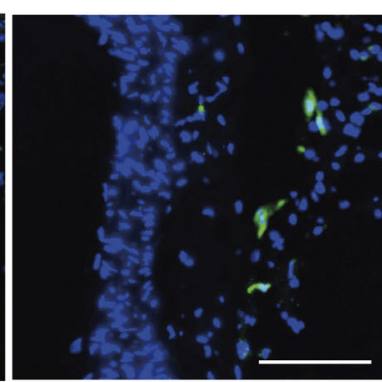

JAK2 ${ }^{\mathrm{V} 617 F-G F P}$

Saline

CD45

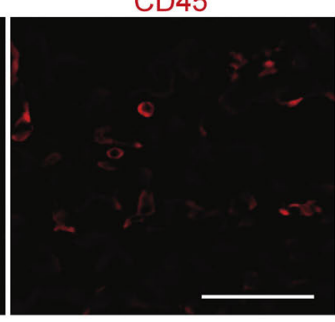

CD68

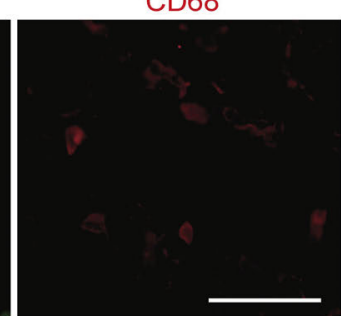

Ly6B.2

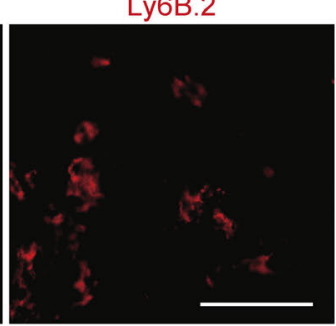

JAK2 ${ }^{\text {V617F-GFP, Ang II }}$
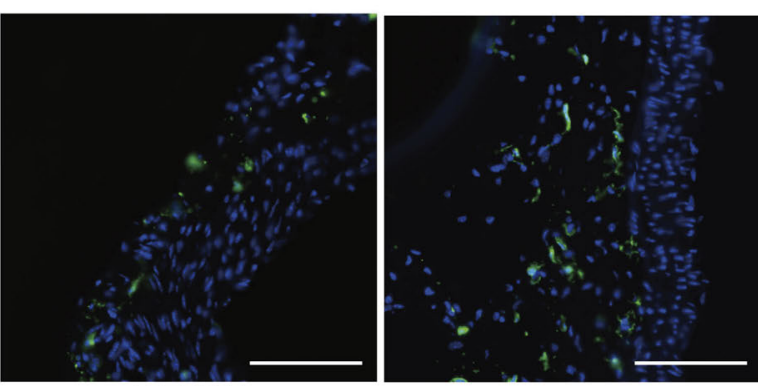

JAK2 ${ }^{\mathrm{V} 617 F}$-GFP

Ang II

DAPI

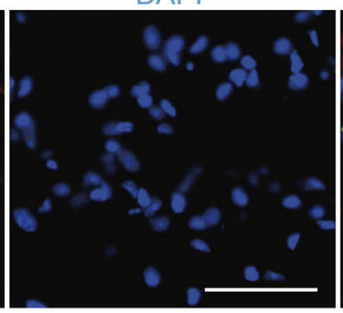

DAPI

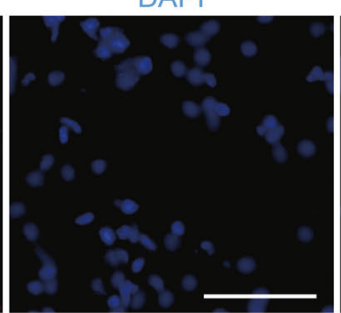

DAPI

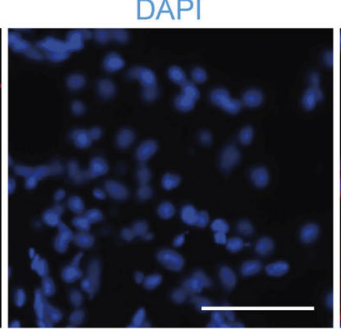

GFP+CD45+DAPI

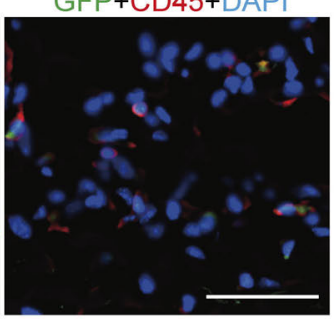

GFP+CD68+DAPI

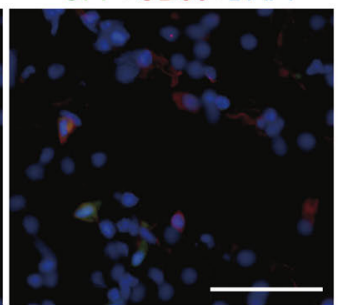

GFP+Ly6B.2+DAP|

Figure 5. Characterization of bone marrow-derived JAK2 V617F hematopoietic cells in the abdominal aorta by use of GFP-transgene. (A) The lethally irradiated $\mathrm{ApoE}^{-/-}$mice were transplanted with bone marrow (BM) cells from the WT/CAG-EGFP (WT-GFP) mice or JAK2 $2^{\text {v617F }} /$ CAG-EGFP (JAK2 ${ }^{\text {v617F }}$-GFP) double transgenic mice. The $\mathrm{ApoE}^{--}$recipient mice were subjected to saline or angiotensin II (Ang II) infusion for 4 weeks. The abdominal aortas were stained with an anti-GFP (green) antibody and DAPI (blue). Scale bars, $100 \mu \mathrm{m}$. (B) Representative immunofluorescence images of the aorta sections stained with anti-CD45 (red), anti-CD68 (red), or anti-Ly6B.2 (red) and anti-GFP (green) antibodies and DAPI (blue) in ApoE ${ }^{-/-}$mice transplanted with JAK ${ }^{2 \mathrm{~V} 617 F}$-GFP BM cells. Scale bars, 50 um. Ang II: angiotensin II; GFP: green fluorescent protein; DAPI: 4',6-diamidino-2-phenylindole; WT: wild-type.

contribute to vascular diseases. In our cohort, $23 \%$ of the patients with JAK2 V617F-positive MPN exhibited the presence of $\mathrm{AA}$. Given that the prevalence of $\mathrm{AA}$ is reported to be $1-5 \%$ in the general population above the age of 65 years, ${ }^{10,11}$ JAK2 V617F-positive MPN might be associated with a higher prevalence of AA. In combination with mouse studies, the presence of JAK2 V617F in leukocytes is likely to be a factor of AA development.

The pathogenetic feature of AA is mediated through the degenerative process involving extracellular matrix remodeling in all layers of the arterial wall. Particularly, local activation of inflammatory responses by immune cells plays an important role in the process. Leukocyte accumulation in inflammatory aneurysms provides addi- tional evidence that inflammation represents variants along a spectrum of aneurysmal disease rather than distinct pathological entities of the inflammatory cells. ${ }^{14} \mathrm{It}$ has been reported that plasma inflammatory cytokine levels in JAK2 V617F-positive MPN are significantly increased in the peripheral blood. ${ }^{42,43}$ Similarly, we showed that circulating JAK2 V617F leukocytes of MPN patients exhibited significant increases in the expression levels of the cytokines of TGFB3 as well as the chemokines of $I L-8$. These findings are supported by previous reports that inflammatory macrophages and neutrophils contributed to AAA development. ${ }^{44,45}$ It has been reported that macrophage NLRP3 inflammation activation in adventitia promotes inflammatory cytokine pro- 
A
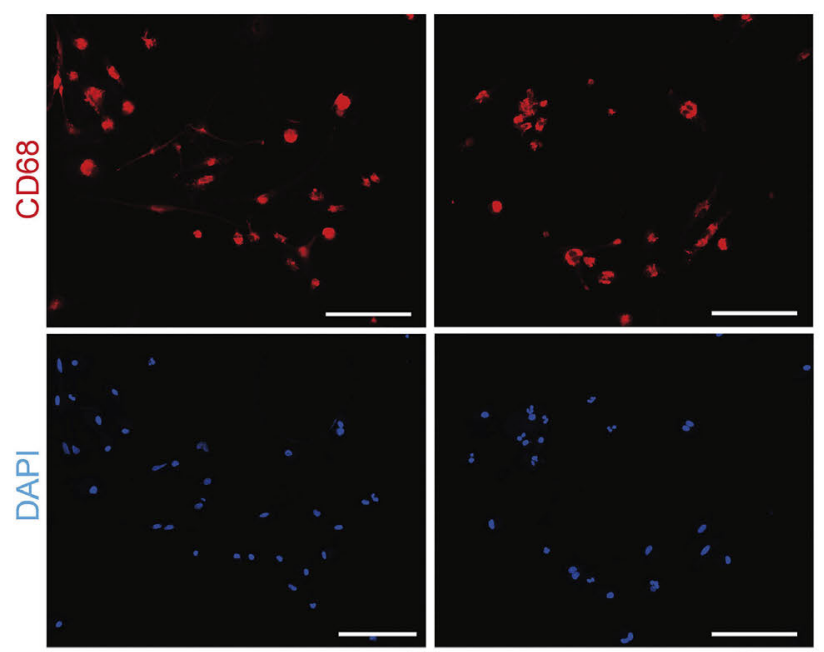

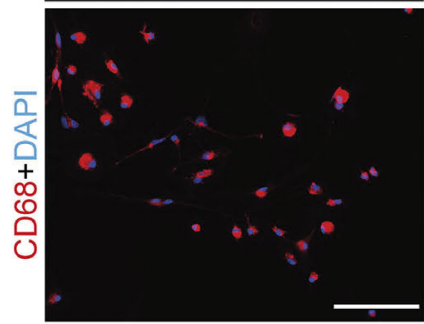

WT

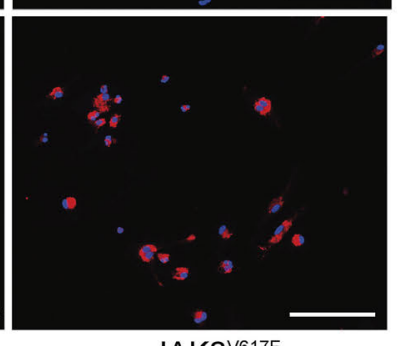

JAK2 $2^{\mathrm{V} 17 \mathrm{~F}}$
B

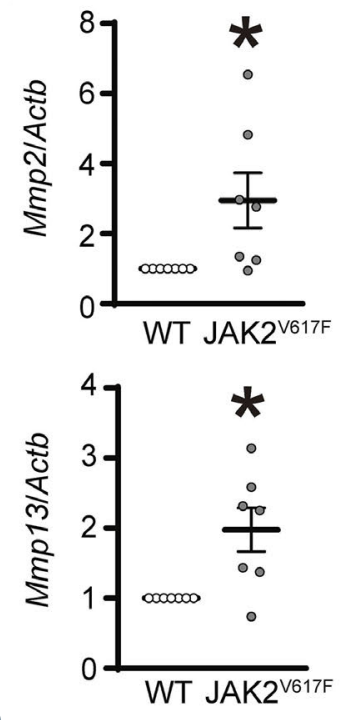

C

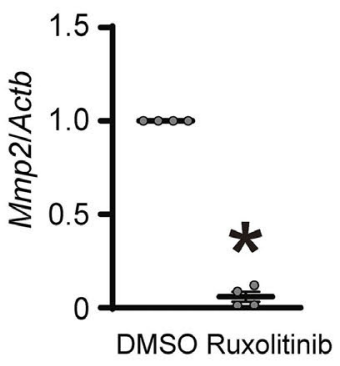

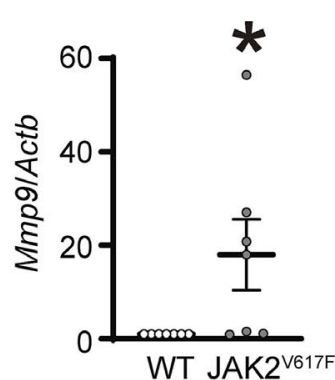

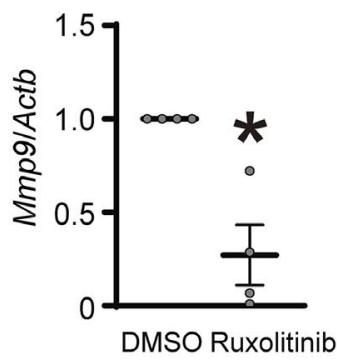

Figure 6. Matrix metalloproteinases are upregulated in bone marrow-derived macrophages with JAK2 V617F expression. (A) Mononuclear cells isolated from bonemarrow (BM) cells of wild-type (WT) mice or JAK2 ${ }^{\mathrm{v} 617 \mathrm{~F}}$ mice were cultured in the presence of granulocyte-macrophage colony stimulating factor for 6 days. Representative immunofluorescence images of the cells stained with anti-CD68 (red) antibody and DAPI (blue). Scale bars, $100 \mu \mathrm{m}$. (B) Relative mRNA expression levels of $M m p 2, M m p 9$, and $M m p 13$ in the cultured mononuclear cells ( $\mathrm{n}=7$ each). (C) Mmp2 and Mmp9 mRNA expressions with and without ruxolitinib pretreatment at $250 \mathrm{nM}$ for 24 hours in the JAK2 ${ }^{\mathrm{v} 17 \mathrm{fF}}$ mononuclear cells $(\mathrm{n}=4$ each). Dimethylsulfoxide (DMSO) was used for control. Actb was used for normalization. The average value for the cultured mononuclear cells from WT mice or DMSO group was set to 1 . All data are presented as mean \pm standard error of the mean. $* P<0.05$ vs. the WT group or DMSO group by the unpaired Student's t-test. JAK2 ${ }^{\text {V617F }}$ JAK2 V617F-expressing transgenic mice.

duction and MMP activation, and contributes to the development of AAA. ${ }^{46}$ Recruitments of macrophages and neutrophils, and expression of pro-inflammatory cytokines may characterize the degenerative process during AA formation. We observed that the JAK2 ${ }^{\mathrm{V} 617 \mathrm{~F}}-\mathrm{BMT}$ mice did not display significant enlargement of the ascending aorta, and therefore it remains to be elucidated whether TAA develops in this model and/or some other condition.

It is known that matrix metalloproteases are closely involved in the pathogenesis of AA through elastin degradation. ${ }^{47}$ Studies have reported that the JAK-STAT signaling pathway is one of the regulators of matrix metalloproteases, ${ }^{48,49}$ and that macrophages are a significant source of matrix metalloproteinases. ${ }^{47,50}$ Both MMP-9 and MMP-2 are required and work in concert to produce AAA. ${ }^{36}$ We have demonstrated here that BM-derived macrophages with JAK2 V617F expression significantly upregulate $M m p 2, M m p 9$, and Mmp13. Likewise, substantially promoted macrophage infiltration was accompanied by increases in MMP-2 activity and pro MMP-9 expression in the abdominal aorta in the Ang II-stimulated JAK2 ${ }^{\mathrm{V} 617 \mathrm{~F}}-\mathrm{BMT}$ mice, suggesting that infiltration of JAK2 V617F-expressing macrophages with activation of matrix metalloproteinases plays causal roles in the patho- genesis of AAA. In agreement, we found significantly higher mRNA levels of $M M P 9$ in the peripheral leukocytes, containing macrophage-progenitor monocytes, of patients with JAK2 V617F-positive MPN compared to control subjects. MMP-9 plasma levels were independently associated with aortic wall thickness and aortic luminal diameter, but not with parameters of atherosclerosis. ${ }^{31}$ Further studies are needed to clarify the MMP-related mechanism for progression to AA in MPN patients.

Currently, ruxolitinib is a clinically available JAK1/2 inhibitor for the treatment of patients with PMF and PV. ${ }^{51}$ Ruxolitinib has been shown to improve long-term survival in patients with PMF and reduced thrombotic events in patients with PV. ${ }^{52-54}$ Given our observations in the present study, ruxolitinib may be useful for preventing the onset and development of AAA in patients with MPN.

It has recently been reported that clonal expansion of hematopoietic cells with somatic mutations, termed as clonal hematopoiesis, is common in the seemingly healthy older general population, and significantly associated with atherosclerotic cardiovascular diseases. ${ }^{55,56}$ Although JAK2 V617F has been identified as one of the recurrent somatic mutations in individuals with clonal hematopoiesis, it remains unclear whether clonal hematopoiesis is involved in AA in addition to stenotic 
atherosclerosis. However, given that our data from murine studies and clinical observations strongly indicate that hematopoietic JAK2 V617F plays crucial roles in the development of AA, we propose that JAK2 V617F-positive clonal hematopoiesis may be also involved in the formation and progression of AA regardless of the hematological phenotypes of MPN. ${ }^{57}$

The limitation of the present study is the small number of patients with MPN included while thrombosis and non-thrombosis categories were also small subsets for
A

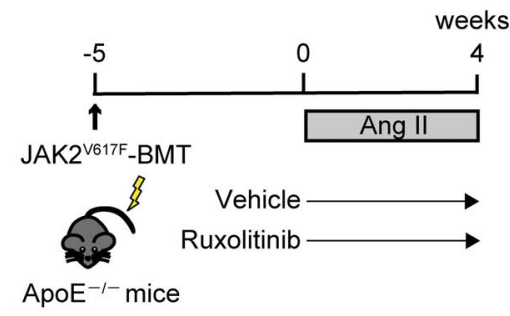

B
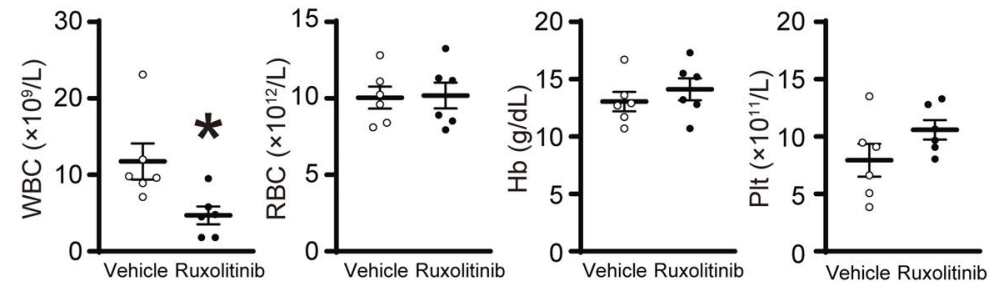

C

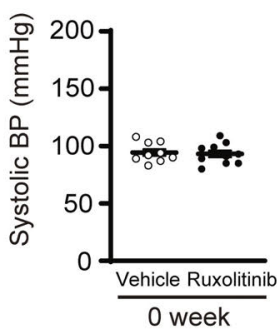

D

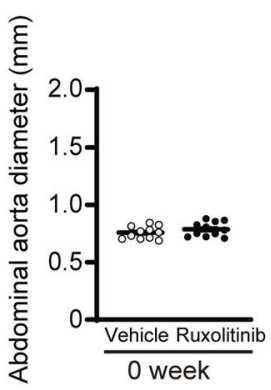

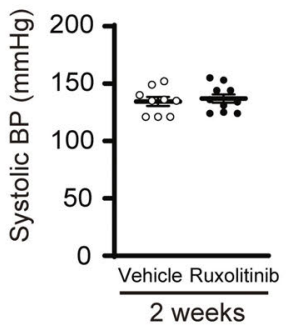

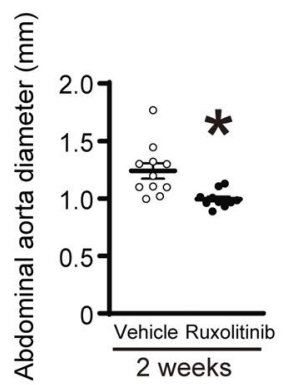

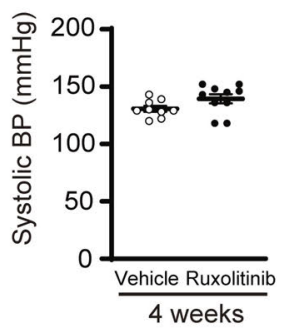

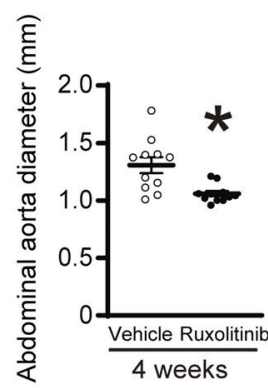

E
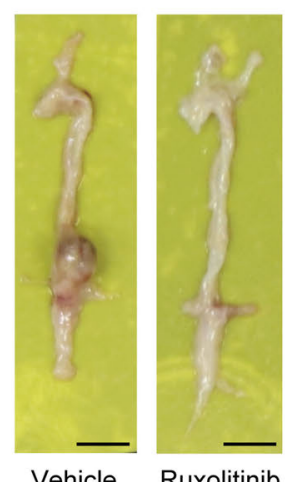

Vehicle Ruxolitinib

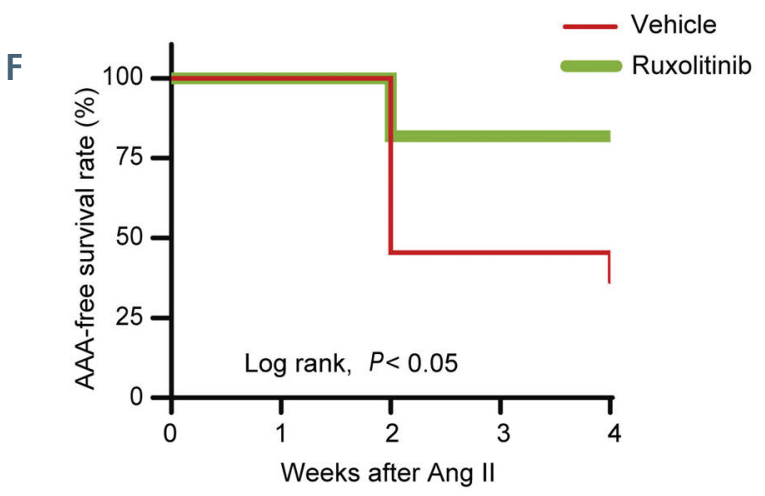

G

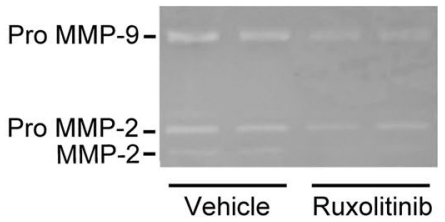

Figure 7. Ruxolitinib attenuates the formation of abdominal aortic aneurysms induced by hematopoietic JAK2 V617F. (A) Schematic diagram of the experimenta design. The ApoE $\%$ recipients transplanted with JAK2 $2^{\mathrm{v} 617 \mathrm{~F}}$ bone marrow cells (JAK2 ${ }^{\mathrm{v} 617 \mathrm{~F}} \mathrm{BMT}$ ) were administered with vehicle (0.5\% methylcellulose) or ruxolitinib was orally at $60 \mathrm{mg} / \mathrm{kg}$ twice daily for 4 weeks along with continuous angiotensin II ( Ang II, $1900 \mathrm{ng} / \mathrm{kg}$ per min) infusion. (B) Blood cell counts at 4 weeks ( $\mathrm{n}=6$ each) and $(C)$ systolic blood pressure (BP) at baseline $(n=9-10), 2$ weeks $(n=9-10)$, and 4 weeks $(n=9-10)$ in the vehicle- or ruxolitinib-treated JAK2 $2^{\text {v617F-BMT mice. }(D)}$ Abdominal aorta diameter at the baseline ( $n=11$ each), 2 weeks ( $n=11$ each), and 4 weeks ( $n=11$ each). (E) Representative images from the aorta. Scale bars, 5

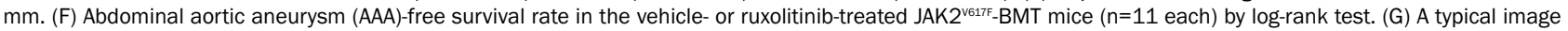
of gelatin zymography using homogenates from abdominal aortas. All data are presented as mean \pm standard error of the mean. $* P<0.05$ vs. the vehicle group by the unpaired Student's $t$-test. WBC: white blood cell count; RBC: red blood cell count; Hb: hemoglobin concentration; PIt: platelet count; BP: blood pressure; MMP: matrix metalloproteinase. 
comparison. Further studies are, thus, needed to validate our findings in larger cohorts.

In conclusion, AA are a possible vascular complication of MPN and hematopoietic JAK2 V617F may causally be involved in the development of AA through macrophage accumulation and MMP activation. The present study also provides novel mechanisms underlying the pathogenesis of AA. Hematopoietic JAK-STAT signaling may be a potential therapeutic target for the development of AA.

\section{Contributions}

$T Y$ and TM designed the research, performed experiments, analyzed the results, and wrote the manuscript; $Y K, K W$, and $K M$ performed the experiments and analyzed the results; KS and TI interpreted the results and supervised the study; SM and $N K$ analyzed the results and interpreted the results, and supervised the study; KI designed and supervised the research, analyzed the data, and wrote the manuscript; YT designed and supervised the research, and approved the final version of the manuscript.

\section{Disclosures}

$T Y$ and KS have received financial support from Janssen Pharmaceutical K.K., Japan; TM has received financial support from Fukuda Denshi Co., Ltd., Japan; Ruxolitinib was provided by Novartis Pharmaceuticals to KI; these companies are, however, not associated with the contents of this study; all other authors declare no conflicts of interest.

\section{Acknowledgments}

The authors thank Ms Tomiko Miura and Ms Shoko Sato, from the Department of Cardiovascular Medicine, Fukushima Medical University, Japan, and Ms Chisato Kubo, from the Office for Gender Equality Support, Fukushima Medical University, Japan, for their technical assistance, as well as Prof. Kazuya Shimoda and Dr. Kotaro Shide, from the Department of Gastroenterology and Hematology, University of Miyazaki, Japan, for providing us with JAK2 $2^{V 617 F}$ mice.

\section{Funding}

This work was supported by JSPS KAKENHI (grant number: JP19K17532) to TY.

\section{References}

1. Kutti J and Ridell B. Epidemiology of the myeloproliferative disorders: essential thrombocythaemia, polycythaemia vera and idiopathic myelofibrosis. Pathol Biol (Paris). 2001;49(2):164-166.

2. Srour SA, Devesa SS, Morton LM, et al. Incidence and patient survival of myeloproliferative neoplasms and myelodysplastic/myeloproliferative neoplasms in the United States, 2001-12. Br J Haematol. 2016;174(3):382-396.

3. Barbui T, Finazzi G and Falanga A. Myeloproliferative neoplasms and thrombosis. Blood. 2013;122(13):2176-2184.

4. De Stefano V, Ghirardi A, Masciulli A, et al. Arterial thrombosis in Philadelphia-negative myeloproliferative neoplasms predicts second cancer: a case-control study. Blood. 2020;135(5):381-386.

5. Hultcrantz M, Björkholm M, Dickman PW, et al. Risk for arterial and venous thrombosis in patients with myeloproliferative neoplasms: a population-based cohort study. Ann Intern Med. 2018;168(5):317-325.

6. Steensma DP, Dewald GW, Lasho TL, et al. The JAK2 V617F activating tyrosine kinase mutation is an infrequent event in both "atypical" myeloproliferative disorders and myelodysplastic syndromes. Blood. 2005;106(4):1207-1209.

7. Shide K, Shimoda HK, Kumano T, et al. Development of ET, primary myelofibrosis and PV in mice expressing JAK2 V617F. Leukemia. 2008;22(1):87-95.

8 . Shide $\mathrm{K}$. The role of driver mutations in myeloproliferative neoplasms: insights from mouse models. Int J Hematol. 2020;111(2):206-216.

9. Wanhainen A, Verzini F, Van Herzeele I, et al. Editor's choice - European Society for Vascular Surgery (ESVS) 2019 clinical practice guidelines on the management of abdominal aorto-iliac artery aneurysms. Eur J Vasc Endovasc Surg. 2019;57(1):8-93.

10. Wanhainen A, Hultgren R, Linne A, et al. Outcome of the swedish nationwide abdominal aortic aneurysm screening program. Circulation. 2016;134(16):1141-1148.

11. Oliver-Williams C, Sweeting MJ, Turton G, et al. Lessons learned about prevalence and growth rates of abdominal aortic aneurysms from a 25-year ultrasound population screening programme. Br J Surg. 2018;105(1):68-74.

12. Gunnarsson K, Wanhainen A, Björck M, Djavani-Gidlund $\mathrm{K}$ and Mani $\mathrm{K}$. Nationwide study of ruptured abdominal aortic aneurysms during twenty years (1994-2013). Ann Surg. 2019 Aug 14. [Epub ahead of print]

13. Dale MA, Ruhlman MK and Baxter BT. Inflammatory cell phenotypes in AAAs: their role and potential as targets for therapy. Arterioscler Thromb Vasc Biol. 2015;35(8):1746-1755.

14. Shimizu K, Mitchell RN and Libby P. Inflammation and cellular immune responses in abdominal aortic aneurysms. Arterioscler Thromb Vasc Biol. 2006;26(5): 987-994.

15. Dutta P, Courties G, Wei Y, et al. Myocardial infarction accelerates atherosclerosis. Nature. 2012;487(7407):325-329.

16. Moazzami K, Lima BB, Hammadah M, et al. Association between change in circulating progenitor cells during exercise stress and risk of adverse cardiovascular events in patients with coronary artery disease. JAMA Cardiol. 2020;5(2):147-155.

17. Barbui T, Finazzi G, Carobbio A, et al. Development and validation of an International Prognostic Score of thrombosis in World Health Organization-essential thrombocythemia (IPSET-thrombosis) Blood. 2012;120(26):5128-5133.

18. Harada-Shirado K, Ikeda K, Ogawa K, et al. Dysregulation of the MIRLET7/HMGA2 axis with methylation of the CDKN2A promoter in myeloproliferative neoplasms. Br J Haematol. 2015;168(3):338-349.

19. Ueda K, Ikeda K, Ikezoe T, et al. Hmga2 collaborates with JAK2V617F in the development of myeloproliferative neoplasms. Blood Adv. 2017;1(15):1001-1015.

20. Piedrahita JA, Zhang SH, Hagaman JR, Oliver PM and Maeda N. Generation of mice carrying a mutant apolipoprotein $\mathrm{E}$ gene inactivated by gene targeting in embryonic stem cells. Proc Natl Acad Sci U S A. 1992;89(10):4471-4475.

21. Okabe M, Ikawa M, Kominami K, Nakanishi $\mathrm{T}$ and Nishimune $\mathrm{Y}$. 'Green mice' as a source of ubiquitous green cells. FEBS Lett. 1997;407(3):313-319.
22. Misaka T, Suzuki S, Miyata M, et al. Deficiency of senescence marker protein 30 exacerbates angiotensin II-induced cardiac remodelling. Cardiovasc Res. 2013;99(3): 461-470.

23. Ortega R, Collado A, Selles F, et al. SGLT-2 (Sodium-Glucose Cotransporter 2) inhibition reduces ang II (Angiotensin II)-induced dissecting abdominal aortic aneurysm in ApoE (Apolipoprotein E) knockout mice. Arterioscler Thromb Vasc Biol. 2019;39(8): 1614-1628.

24. Patel J, Douglas G, Kerr AG, Hale AB and Channon KM. Effect of irradiation and bone marrow transplantation on angiotensin II-induced aortic inflammation in ApoE knockout mice. Atherosclerosis. 2018;276:74-82.

25. Quintás-Cardama A, Vaddi K, Liu P, et al. Preclinical characterization of the selective JAK1/2 inhibitor INCB018424: therapeutic implications for the treatment of myeloproliferative neoplasms. Blood. 2010;115(15): 3109-3117.

26. Lee S, Shah T, Yin C, et al. Ruxolitinib significantly enhances in vitro apoptosis in Hodgkin lymphoma and primary mediastinal B-cell lymphoma and survival in a lymphoma xenograft murine model Oncotarget. 2018;9(11):9776-9788.

27. Hiratzka LF, Bakris GL, Beckman JA, et al. 2010 ACCF/AHA/AATS/ACR/ASA/SCA/ SCAI/SIR/STS/SVM guidelines for the diagnosis and management of patients with Thoracic Aortic Disease: a report of the American College of Cardiology Foundation/American Heart Association Task Force on Practice Guidelines, American Association for Thoracic Surgery, American College of Radiology, American Stroke Association, Society of Cardiovascular Anesthesiologists, Society for Cardiovascular Angiography and Interventions, Society of Interventional Radiology, Society of Thoracic Surgeons, and Society for Vascular Medicine. Circulation. 2010;121(13):e266-369.

28. Carobbio A, Thiele J, Passamonti F, et al. Risk factors for arterial and venous thrombosis in WHO-defined essential thrombocythemia: an international study of 891 patients. Blood. 2011;117(22):5857-5859.

29. Barbui T, Tefferi A, Vannucchi AM, et al. 
Philadelphia chromosome-negative classical myeloproliferative neoplasms: revised management recommendations from European LeukemiaNet. Leukemia. 2018; 32(5):1057-1069

30. Lindholt JS and Shi GP. Chronic inflammation, immune response, and infection in abdominal aortic aneurysms. Eur J Vasc Endovasc Surg. 2006;31(5):453-463.

31. Grodin JL, Powell-Wiley TM, Ayers CR, et al. Circulating levels of matrix metalloproteinase- 9 and abdominal aortic pathology: from the Dallas Heart Study. Vasc Med. 2011;16(5):339-345.

32. Tefferi A, Nicolosi $M$, Penna $D$, et al. Development of a prognostically relevant cachexia index in primary myelofibrosis using serum albumin and cholesterol levels. Blood Adv. 2018;2(15):1980-1984.

33. Fujita H, Hamaki T, Handa N, Ohwada A, Tomiyama J and Nishimura S. Hypocholesterolemia in patients with polycythemia vera. J Clin Exp Hematop. 2012;52(2):85-89.

34. Deng GG, Martin-McNulty B, Sukovich DA, et al. Urokinase-type plasminogen activator plays a critical role in angiotensin II-induced abdominal aortic aneurysm. Circ Res. 2003;92(5):510-517.

35. Satoh K, Nigro P, Matoba T, et al. Cyclophilin A enhances vascular oxidative stress and the development of angiotensin II-induced aortic aneurysms. Nat Med. 2009;15(6):649-656.

36. Longo GM, Xiong W, Greiner TC, Zhao Y, Fiotti N and Baxter BT. Matrix metalloproteinases 2 and 9 work in concert to produce aortic aneurysms. J Clin Invest. 2002;110 (5):625-632.

37. Quintana RA and Taylor WR. Cellular mechanisms of aortic aneurysm formation. Circ Res. 2019;124(4):607-618.

38. Hernández Ríos M, Sorsa T, Obregón F, et al. Proteolytic roles of matrix metalloproteinase (MMP)-13 during progression of chronic periodontitis: initial evidence for MMP-13/MMP-9 activation cascade. J Clin Periodontol. 2009;36(12):1011-1017.
39. Edelmann B, Gupta N, Schnoeder TM, et al. JAK2-V617F promotes venous thrombosis through $\beta 1 / \beta 2$ integrin activation. J Clin Invest. 2018;128(10):4359-4371.

40. Barbui T, Barosi G, Birgegard G, et al Philadelphia-negative classical myeloproliferative neoplasms: critical concepts and management recommendations from European LeukemiaNet. J Clin Oncol. 2011;29(6):761-770.

41. Campbell PJ, MacLean C, Beer PA, et al Correlation of blood counts with vascular complications in essential thrombocythemia: analysis of the prospective PT1 cohort. Blood. 2012;120(7):1409-1411.

42. Wehrle J, Seeger TS, Schwemmers S, Pfeifer D, Bulashevska A and Pahl HL. Transcription factor nuclear factor erythroid-2 mediates expression of the cytokine interleukin 8, a known predictor of inferior outcome in patients with myeloproliferative neoplasms. Haematologica. 2013;98(7):1073-1080.

43. Dutta A, Hutchison RE and Mohi G. Hmga2 promotes the development of myelofibrosis in Jak2(V617F) knockin mice by enhancing TGF- $\beta 1$ and Cxcl12 pathways. Blood. 2017;130(7):920-932

44. Hadi T, Boytard L, Silvestro M, et al. Macrophage-derived netrin-1 promotes abdominal aortic aneurysm formation by activating MMP3 in vascular smooth muscle cells. Nat Commun. 2018;9:5022.

45. Eliason JL, Hannawa KK, Ailawadi G, et al Neutrophil depletion inhibits experimental abdominal aortic aneurysm formation. Circulation. 2005;112(2):232-240.

46. Usui F, Shirasuna K, Kimura $\mathrm{H}$, et al. Inflammasome activation by mitochondrial oxidative stress in macrophages leads to the development of angiotensin II-induced aortic aneurysm. Arterioscler Thromb Vasc Biol. 2015;35(1):127-136.

47. Wang Y, Ait-Oufella $\mathrm{H}$, Herbin $\mathrm{O}$, et al. TGF-beta activity protects against inflammatory aortic aneurysm progression and complications in angiotensin II-infused mice. J Clin Invest. 2010;120(2):422-432.
48. Li WQ, Dehnade F and Zafarullah $M$. Oncostatin M-induced matrix metalloproteinase and tissue inhibitor of metalloproteinase-3 genes expression in chondrocytes requires Janus kinase/STAT signaling pathway. J Immunol. 2001;166(1):3491-3498.

49. Ghosh A, Pechota A, Coleman D, Upchurch GR, Jr. and Eliason JL. Cigarette smoke-induced MMP2 and MMP9 secretion from aortic vascular smooth cells is mediated via the Jak/Stat pathway. Hum Pathol. 2015;46(2):284-294.

50. Kothari P, Pestana R, Mesraoua R, et al. IL6-mediated induction of matrix metalloproteinase- 9 is modulated by JAK-dependent IL-10 expression in macrophages. Immunol. 2014;192(1):349-357.

51. Harrison C, Kiladjian JJ, Al-Ali HK, et al JAK inhibition with ruxolitinib versus best available therapy for myelofibrosis. $N$ Engl J Med. 2012;366(9):787-798.

52. Vannucchi AM and Harrison CN. Emerging treatments for classical myeloproliferative neoplasms. Blood. 2017;129(6):693-703.

53. Harrison CN, Vannucchi AM, Kiladiian JJ, et al. Long-term findings from COMFORTII, a phase 3 study of ruxolitinib vs best available therapy for myelofibrosis. Leukemia. 2016;30(8):1701-1707.

54. Verstovsek S, Vannucchi AM, Griesshammer M, et al. Ruxolitinib versus best available therapy in patients with polycythemia vera: 80-week follow-up from the RESPONSE trial. Haematologica. 2016;101(7):821-829.

55. Jaiswal S, Natarajan P, Silver AJ, et al Clonal hematopoiesis and risk of atherosclerotic cardiovascular disease. $\mathrm{N}$ Engl J Med. 2017;377(2):111-121.

56. Mas-Peiro S, Hoffmann J, Fichtlscherer S, et al. Clonal haematopoiesis in patients with degenerative aortic valve stenosis undergoing transcatheter aortic valve implantation. Eur Heart J. 2020;41(8):933-939.

57. Jaiswal S, Fontanillas P, Flannick J, et al Age-related clonal hematopoiesis associated with adverse outcomes. N Engl J Med. 2014;371(26):2488-2498. 